\title{
Optimization and Large Scale Computation of an Entropy-Based Moment Closure*
}

\author{
C. Kristopher Garrett ${ }^{\dagger} \quad$ Cory Hauck ${ }^{\ddagger} \quad$ Judith Hill ${ }^{\S}$
}

July 13, 2015

${ }^{*}$ The submitted manuscript has been authored by a contractor of the U.S. Government under Contract No DE-AC05-00OR22725. Accordingly, the U.S. Government retains a non-exclusive, royalty-free license to publish or reproduce the published form of this contribution, or allow others to do so, for U.S. Government purposes.

${ }^{\dagger}$ Computer Science and Mathematics Division, Oak Ridge National Laboratory, Oak Ridge, TN. E-mail: garrettck@ornl.gov.

${ }^{\ddagger}$ Computer Science and Mathematics Division, Oak Ridge National Laboratory, Oak Ridge, TN \& Department of Mathematics, University of Tennessee, Knoxville, TN. E-mail: hauckc@ornl.gov.

${ }^{\S}$ Oak Ridge Leadership Computing Facility, Oak Ridge National Laboratory, Oak Ridge, TN. E-mail: hilljcœornl.gov. 
particle concentrations, especially in the streaming particle regime, where collisions are rare [7]. An alternative to the $\mathrm{P}_{N}$ closure is a more complicated nonlinear closure based on minimizing a physically relevant, convex function related to the entropy of the physical system [13,20,31]. The resulting method, colloquially termed $\mathrm{M}_{N}$ in the radiation transport community, yields a nonlinear hyperbolic balance law that, unlike the $\mathrm{P}_{N}$ method, formally captures the correct streaming limit [13], but at the same time, is accurate in scattering dominated regimes $[9,17]$. The $\mathrm{M}_{N}$ method has been shown to be more accurate in several test cases $[8,14,16,20]$ and in several different applications $[6,15,18,33,36,45]$. However, it requires the solution of a minimization problem at every spatial cell of the discretized domain. In general, the minimization must be solved numerically. This causes the $M_{N}$ method to require many more floating point operations than the $\mathrm{P}_{N}$ method, even though the methods have the same data communication patterns. To avoid the computational overhead of the minimization problem, some approaches generate approximations of the entropybased closure using look-up tables or interpolation schemes [34,35,47]. Such approximations are generally less robust than the original entropy-based closures and have, so far, been limited to low-order moment systems. Even so, in some cases, they maintain enough of the structure from the entropy-based approach to be considered as a suitable alternative.

The computational expense of the $\mathrm{M}_{N}$ method makes it prohibitive for serial or even small-scale parallel implementations. However, for large scale computations on high performance machines, it is expected that the computing time for the $\mathrm{P}_{N}$ method will eventually become dominated by communication, and in such cases, the $\mathrm{M}_{N}$ method will be more competitive in time to solution. Therefore progress in this area depends on three factors: (i) algorithmic improvements in solving the minimization problem that defines the $\mathrm{M}_{N}$ closure, (ii) performance improvements that leverage the available computer hardware, and (iii) scaling to extremely large problems. The work here builds on algorithmic improvements in $[2,3] .{ }^{1}$ In the current paper, we address the other two factors. First we design and test several optimizations for the $\mathrm{M}_{N}$ algorithm that reduce the time to solution by as much as 10 times in some cases. We then explore scalability of the $\mathrm{M}_{N}$ algorithm using an explicit time integration algorithm. Using the supercomputer Titan, which is housed at Oak Ridge National Laboratory and operated by the Oak Ridge Leadership Computing Facility, we find the $\mathrm{M}_{N}$ algorithm weakly scales almost perfectly out to 17,576 compute nodes while the $\mathrm{P}_{N}$ algorithm displays an increase in time per node by a factor of $1.2 \mathrm{x}$ to $4 \mathrm{x}$, depending on the amount of data per node. However, even with performance improvements, the time to solution of the $\mathrm{M}_{N}$ algorithm is still approximately 25 times greater than the time to solution of the $\mathrm{P}_{N}$ algorithm when both are run at full scale.

The layout of the paper is as follows. In Section 2, we briefly summarize the moment approach, discuss important implementation details, and introduce two test problems that will be used for numerical simulations. In Section 3, we introduce three improvements to the $M_{N}$ algorithm: one that leverages structure in the Hessian matrix of the $\mathrm{M}_{N}$ minimization algorithm and two that use GPUs to accelerate the two most arithmetically intensive parts of the computation. In Section 4, $\mathrm{M}_{N}$ statistics and timing results are presented for the two test problems. Results of weak scaling tests for $\mathrm{P}_{N}$ and $\mathrm{M}_{N}$ are also compared. Section 5 is for conclusions and discussion. The Appendix contains useful technical details about spherical harmonics and Gaunt coefficients. Section D of the Appendix contains a glossary for the variables used throughout the paper.

\footnotetext{
${ }^{1}$ Although not discussed here, other efforts to solve the minimization problem have been documented in [3].
} 


\section{Moment equations}

In this section, we briefly summarize the necessary background material on moment methods, give details on numerical implementation, and present two initial conditions used in the numerical examples.

\section{$2.1 \quad$ Formulation}

The governing equation for this study is a linear kinetic transport equation for unit speed particles in an infinite medium. This equation takes the form

$$
\partial_{t} f+\Omega \cdot \nabla_{x} f=\frac{1}{4 \pi} \sigma_{\mathrm{s}}\langle f\rangle-\sigma_{\mathrm{t}} f,
$$

where (i) $x \in \mathbb{R}^{3}$ is a point in space, (ii) $\Omega \in \mathbb{S}^{2}$ (the unit sphere) is a velocity direction (velocity magnitude is 1 ), (iii) $t>0$ is a point in time, (iv) $f(x, \Omega, t)$ is the kinetic density of particles with respect to the measure $d x d \Omega,(\mathrm{v}) \sigma_{\mathrm{s}}(x)$ is the scattering cross section, (vi) $\sigma_{\mathrm{t}}(x)$ is the total cross section, and (vii) $\langle\cdot\rangle=\int_{\mathbb{S}^{2}} \cdot d \Omega$. In general $\sigma_{\mathrm{t}} \geq \sigma_{\mathrm{s}} \geq 0$; for the purposes of this paper, we set $\sigma_{\mathrm{t}}=\sigma_{\mathrm{s}}=1$.

Moment equations are derived from (1). Let $\mathbf{m}(\Omega)=\left(m_{1}(\Omega), \ldots, m_{M}(\Omega)\right)^{T}$ be a finite vector of real-valued, normalized spherical harmonics ${ }^{2}$ (defined in Appendix A) of degree less than or equal to $N$ with length $M=(N+1)^{2}$. Define the finite vector of moments with respect to $\Omega$ as $\mathbf{u}_{f}(x, t)=\langle\mathbf{m} f\rangle$. Then according to $(1), \mathbf{u}_{f}$ satisfies

$$
\partial_{t} \mathbf{u}_{f}+\nabla_{x} \cdot\langle\Omega \mathbf{m} f\rangle=-Q \mathbf{u}_{f},
$$

where $Q=\operatorname{diag}(0,1, \ldots, 1)$. The system (2) is not closed because the flux $\langle\Omega \mathbf{m} f\rangle$ is a linear combination of moments up to degree $N+1$ whereas $\mathbf{u}_{f}$ only contains moments up to and including degree $N$.

The system (2) is closed using an expansion operator

$$
\mathcal{E}: D \ni \mathbf{v} \longmapsto \mathcal{E}(\mathbf{v}) \in L^{1}\left(\mathbb{S}^{2}\right),
$$

where $D \subset \mathbb{R}^{M}$, and $\mathcal{E}$ satisfies the compatibility condition $\mathbf{v}=\langle\mathbf{m} \mathcal{E}(\mathbf{v})\rangle \cdot{ }^{3}$ Approximating $f$ by $\mathcal{E}(\mathbf{u})$ in $(2)$ yields the closed system

$$
\partial_{t} \mathbf{u}+\nabla_{x} \cdot\langle\Omega \mathbf{m} \mathcal{E}(\mathbf{u})\rangle=-Q \mathbf{u}
$$

where $\mathbf{u}$ approximates the true moments $\mathbf{u}_{f}$. Equation (4) may be equivalently viewed as the following nonlinear Galerkin approximation: Find $\mathbf{u} \in D$ such that

$$
\partial_{t}\langle\phi \mathcal{E}(\mathbf{u})\rangle+\nabla_{x} \cdot\langle\Omega \phi \mathcal{E}(\mathbf{u})\rangle=-Q\langle\phi \mathcal{E}(\mathbf{u})\rangle,
$$

for all $\phi \in \operatorname{span}\left\{m_{1}, \ldots, m_{M}\right\}$.

In this paper, we focus on the entropy-based moment method $\mathrm{M}_{N}$, but for the purposes of comparison, we also consider the classical moment method $\mathrm{P}_{N}$. Below are their expansion operators $[16]$ :

\footnotetext{
${ }^{2}$ The choice of spherical harmonics is not necessary, but it is common in radiation transport. This is because they are eigenfunctions of a more general scattering operator [32].

${ }^{3}$ For the $\mathrm{P}_{N}$ equations, the domain $D$ of the expansion operator $\mathcal{E}$ is all of $\mathbb{R}^{M}$. For the $\mathrm{M}_{N}$ equations $D=$ $\left\{\mathbf{w} \in \mathbb{R}^{M}: \mathbf{w}=\langle\mathbf{m} F\rangle\right.$ for some nonnegative $\left.F \in L^{1}\left(\mathbb{S}^{2}\right)\right\}$ is the set of realizable moments. More information on realizability in the context of kinetic equations can be found in [21,23,43]. For more general theory, see [10,24,44].
} 
- $\mathrm{P}_{N}: \mathcal{E}(\mathbf{u})=\mathbf{u}^{T}\left\langle\mathbf{m} \mathbf{m}^{T}\right\rangle^{-1} \mathbf{m}=\mathbf{u}^{T} \mathbf{m} ;{ }^{4}$

- $\mathrm{M}_{N}: \mathcal{E}(\mathbf{u})=\exp \left(\hat{\boldsymbol{\alpha}}(\mathbf{u})^{T} \mathbf{m}\right)$, where $\hat{\boldsymbol{\alpha}}: \mathbb{R}^{M} \rightarrow \mathbb{R}^{M}$ is given by

$$
\hat{\boldsymbol{\alpha}}=\underset{\boldsymbol{\alpha} \in \mathbb{R}^{M}}{\arg \min }\left\langle\exp \left(\boldsymbol{\alpha}^{T} \mathbf{m}\right)\right\rangle-\boldsymbol{\alpha}^{T} \mathbf{u} .
$$

\subsection{Implementation}

In this section, we present an overview of several implementation details including (i) quadrature approximation in angle, (ii) finite volume discretization in space, (iii) solving the $\mathrm{M}_{N}$ minimization problem (6), and (iv) time discretization method. Since many indices and variables are used, a glossary is available in Appendix D for reference.

Quadrature approximation. For various parts of the $\mathrm{M}_{N}$ implementation, we use a quadrature to approximate integrals with respect to $\Omega$ over the domain $\mathbb{S}^{2}$. To this end, we use a product quadrature on the sphere $[4,46]$ with $n_{g}$ Gauss-Legendre nodes/weights on the $\Omega_{3}$-axis and $2 n_{g}$ equally spaced nodes/weights around latitudinal circles of the sphere for a total of $Q=2 n_{g}^{2}$ quadrature nodes/weights. This quadrature has the property that it integrates spherical harmonics of degree less than or equal to $2 n_{g}-1$ exactly. We use the notation $\left(\Omega_{q}, w_{q}\right)$ to represent the quadrature nodes and weights, respectively, for $q=1, \ldots, Q$. For any $G \in L^{1}\left(\mathbb{S}^{2}\right)$, integrals will be approximated as

$$
\int_{\mathbb{S}^{2}} G(\Omega) d \Omega \approx \sum_{q=1}^{Q} w_{q} G\left(\Omega_{q}\right) .
$$

Several other quadratures exist that can integrate spherical harmonics of the same degree as the cross-product quadrature, but with fewer points. These include quadratures by Lebedev [26-30] and by Ahrens and Beylkin [1]. As the number of quadrature points increases, these quadratures are optimal in the number of nodes/weights and asymptotically use $2 / 3$ the number of points that the product quadrature uses. See [5] for a general discussion. While we use the product quadrature for simplicity, these more efficient quadratures will likely yield a commensurate improvement in overall run-time of the $\mathrm{M}_{N}$ simulations.

Spatial discretization. For the spatial discretization, we use a finite volume method. In particular, we use a kinetic scheme $[12,19,38,39]$. This scheme is derived by first spatially discretizing the kinetic equation (1) using a finite volume approach and then taking moments of the discretization and applying the closure.

More specifically, we use a uniform cartesian grid with cells $C_{i j k}$ and cell sizes $\Delta x \times \Delta y \times \Delta z$. For compactness, define

$$
\begin{aligned}
\boldsymbol{\delta}_{i j k}:= & \frac{1}{\Delta x}\left\langle\Omega_{1} \mathbf{m}\left(\mathcal{E}_{i+1 / 2, j, k}-\mathcal{E}_{i-1 / 2, j, k}\right)\right\rangle+\frac{1}{\Delta y}\left\langle\Omega_{2} \mathbf{m}\left(\mathcal{E}_{i, j+1 / 2, k}-\mathcal{E}_{i, j-1 / 2, k}\right)\right\rangle+ \\
& \frac{1}{\Delta z}\left\langle\Omega_{3} \mathbf{m}\left(\mathcal{E}_{i, j, k+1 / 2}-\mathcal{E}_{i, j, k-1 / 2}\right)\right\rangle,
\end{aligned}
$$

\footnotetext{
${ }^{4}$ In general, the matrix $\left\langle\mathbf{m m}^{T}\right\rangle$ is diagonal since the real-valued spherical harmonics are an orthogonal basis. Furthermore, by normalizing the spherical harmonics as we do in this paper, $\left\langle\mathbf{m m}^{T}\right\rangle$ is the identity matrix.
} 


\footnotetext{
${ }^{5}$ The algorithm in [2] uses an adaptive quadrature and a regularization procedure for ill-conditioned problems. Neither of these were required for the problems in this paper.
}

where $\mathcal{E}_{i \pm 1 / 2, j, k}$ approximate the average of $\mathcal{E}(\mathbf{u})$ on the two boundary faces of $C_{i j k}$ perpendicular to the $x$-axis, and $\mathcal{E}_{i, j \pm 1 / 2, k}$ and $\mathcal{E}_{i, j, k \pm 1 / 2}$ are defined similarly. Then, the spatial discretization is given by

$$
\partial_{t} \mathbf{u}_{i j k}+\boldsymbol{\delta}_{i j k}+Q \mathbf{u}_{i j k}=0
$$

where $\mathbf{u}_{i j k}$ is an approximation of the corresponding cell average for $\mathbf{u}$.

We use a second-order upwind scheme to calculate edge values for both $\mathrm{P}_{N}$ and $\mathrm{M}_{N}$. For the $\mathrm{P}_{N}$ closure, the integrals in (8) can be computed exactly without explicitly calculating the edge values $\mathcal{E}_{i \pm 1 / 2, j, k}, \mathcal{E}_{i, j \pm 1 / 2, k}$, and $\mathcal{E}_{i, j, k \pm 1 / 2}$. However, for the $\mathrm{M}_{N}$ closure, a quadrature rule is required to approximate the integrals. In addition, a double minmod limiter is used to limit slopes for $\mathrm{M}_{N}$ to ensure realizable moments as in [2,16]. More details about the discretization for both $\mathrm{P}_{N}$ and $\mathrm{M}_{N}$ can be found in [16], where the same discretization approach was used in a two-dimensional spatial setting. (Note that we abuse the notation $\mathcal{E}_{i \pm 1 / 2, j, k}, \mathcal{E}_{i, j \pm 1 / 2, k}, \mathcal{E}_{i, j, k \pm 1 / 2}$, and $\boldsymbol{\delta}_{i j k}$, which may refer to values for either $\mathrm{P}_{N}$ or $\mathrm{M}_{N}$, although the two methods yield different results.)

Minimization problem for $\mathbf{M}_{N}$. Given $\mathbf{u}$, the objective function for the minimization problem (6) is

$$
\Phi(\boldsymbol{\alpha} ; \mathbf{u})=\int_{\mathbb{S}^{2}} \exp \left(\boldsymbol{\alpha}^{T} \mathbf{m}\right) d \Omega-\boldsymbol{\alpha}^{T} \mathbf{u}
$$

We use the Newton-type solver developed in [2], which requires the gradient and Hessian of $\Phi$ :

$$
\mathbf{g}(\boldsymbol{\alpha})=\left\langle\mathbf{m} e^{\boldsymbol{\alpha}^{T} \mathbf{m}}\right\rangle-\mathbf{u} \quad \text { and } \quad H(\boldsymbol{\alpha})=\left\langle\mathbf{m m}^{T} e^{\boldsymbol{\alpha}^{T} \mathbf{m}}\right\rangle .
$$

A summary of the minimization algorithm is given in Algorithm 1. For full details consult $[2]^{5}$.

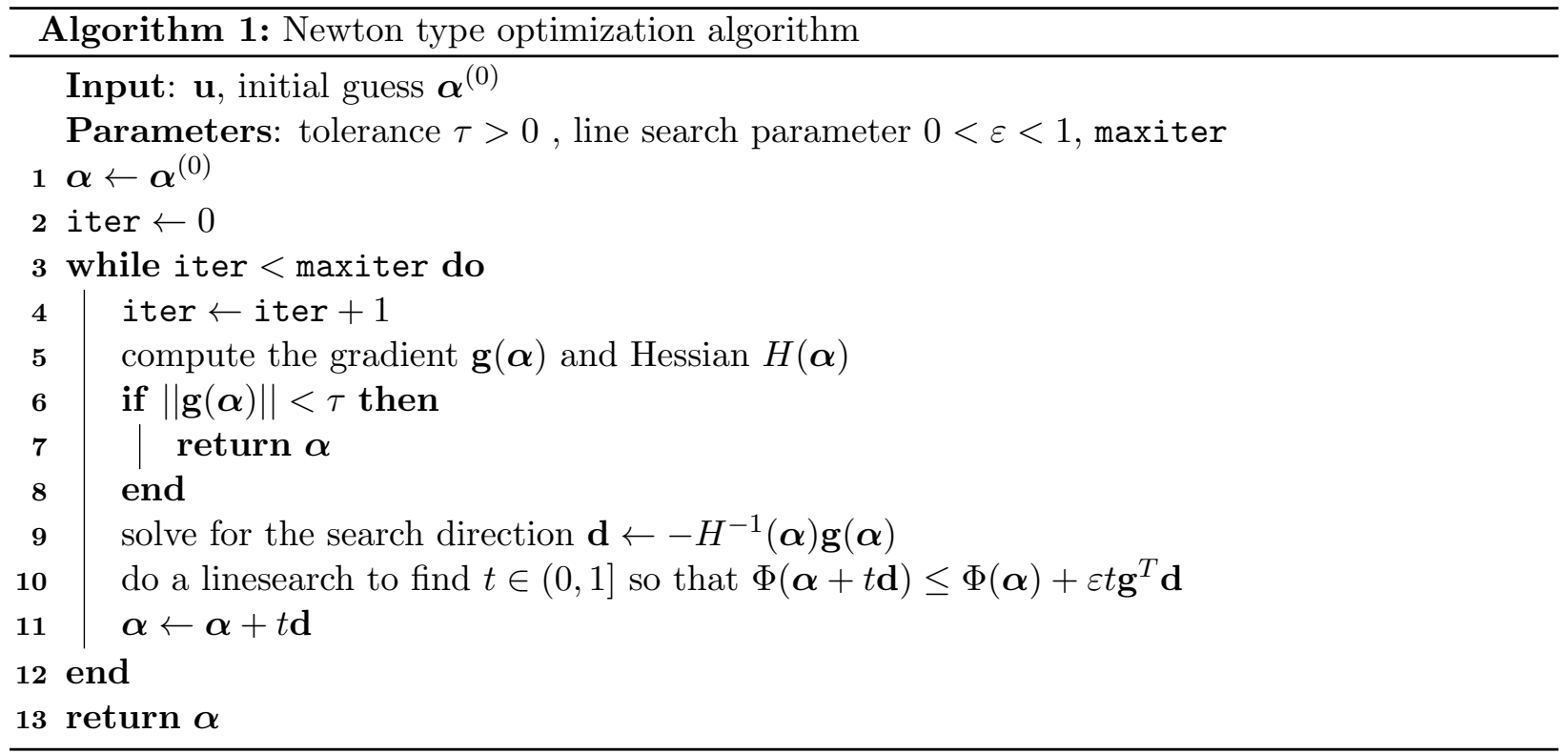

Since $H$ is symmetric and positive definite, $\mathbf{d}$ is computed using a Cholesky decomposition. In the linesearch, we use $\varepsilon=10^{-3}, \tau=10^{-4}$, and maxiter $=100$. In our simulations, iter never reached the limit maxiter. Typically, iter took on values of only 1 or 2 , but a few minimizations required values as high as 25 . 
One important implementation detail is the calculation of the objective $\Phi$ and the gradient g, both of which can be computed from the Hessian. Given the value of the Hessian $H$, the computation of $\Phi$ and $\mathbf{g}$ are trivially computed since $m_{1}$ is a constant and thus

$$
\Phi=\frac{1}{m_{1}^{2}} H_{1,1}-\boldsymbol{\alpha}^{T} \mathbf{u} \quad \text { and } \quad \mathbf{g}=\frac{1}{m_{1}} H_{:, 1}-\mathbf{u},
$$

where $H_{:, 1}$ indicates the first column of $H$ and $H_{1,1}$ is the $(1,1)$ entry of $H$. Hence, calculation of the Hessian is the largest computational cost of the minimization algorithm.

Time discretization. We use the explicit Heun's method (SSP-RK2) for time integration which is 2 nd order, and under the time step constraint

$$
\Delta t \leq \frac{1}{2\left(\frac{1}{\Delta x}+\frac{1}{\Delta y}+\frac{1}{\Delta z}\right)+\sigma_{t}},
$$

ensures realizability for the $\mathrm{M}_{N}$ closure $[2]^{6}$.

Algorithms 2 and 3 outline the approach used to calculate one time step for the $\mathrm{M}_{N}$ and $\mathrm{P}_{N}$ algorithms, respectively. The code uses one MPI task per compute node, with OpenMP or CUDA used to parallelize computations on each compute node. The four functions from lines $3-6$ do the following

- comm - communicates boundary data between compute nodes via MPI;

- min - implements Algorithm 1 ( $\mathrm{M}_{N}$ only);

- flux_pn/flux_mn - calculates $\delta$ from (8) for $\mathrm{P}_{N}$ and $\mathrm{M}_{N}$, respectively;

- euler - computes $\mathbf{u}^{(s)}=\mathbf{u}^{(s-1)}-\Delta t \boldsymbol{\delta}-\Delta t Q \mathbf{u}^{(s-1)}$, where $s$ is the Runge-Kutta stage of the Heun method.
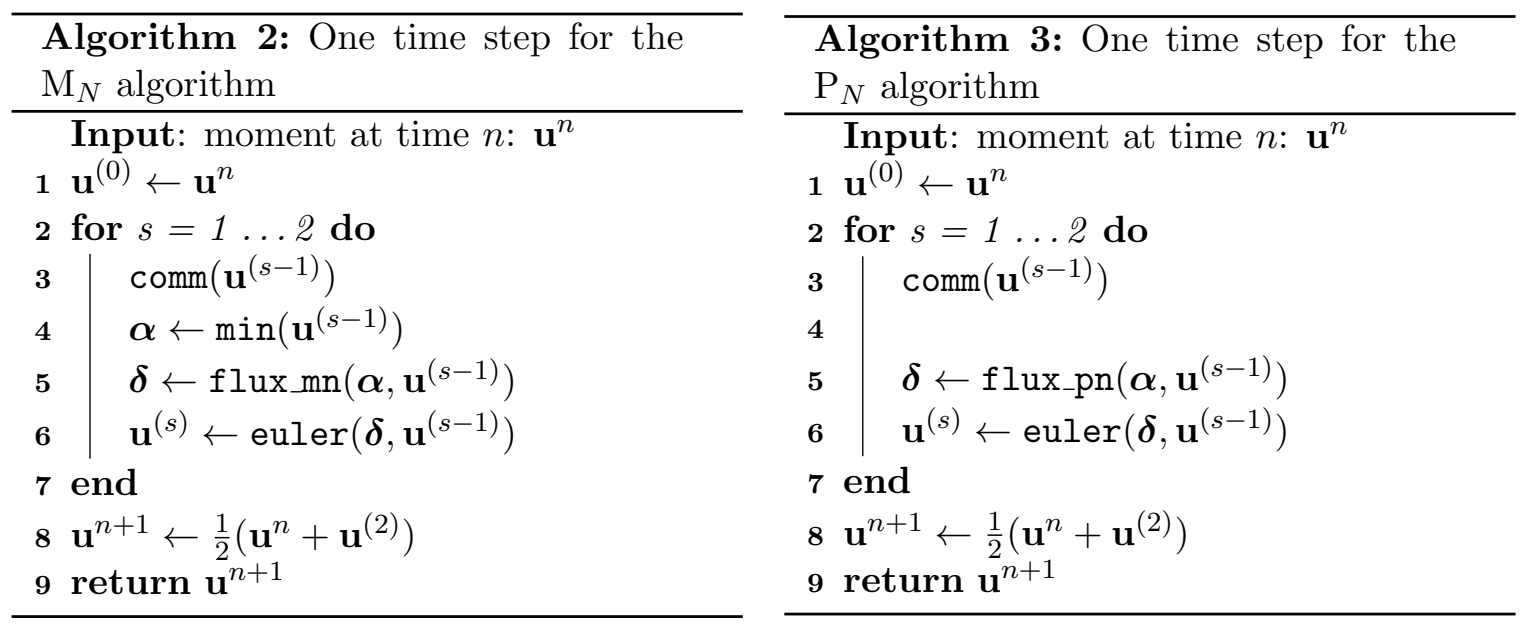

\footnotetext{
${ }^{6}$ This is only proven for 1D in space in the reference, but the extension to higher dimensions is straightforward.
} 


\subsection{Test problems}

Two initial conditions were used for the tests we performed: a "smooth" initial condition

$$
u_{\ell}^{\mathrm{S}}= \begin{cases}2 \sqrt{\pi}(2+\cos (2 \pi x) \cos (2 \pi y) \cos (2 \pi z)), & \ell=1 \\ 0.3 u_{1}, & \ell=2,3,4 \\ 0, & \ell>4\end{cases}
$$

and a narrow "Gaussian" initial condition:

$$
u_{\ell}^{\mathrm{G}}= \begin{cases}2 \sqrt{\pi} \max \left\{\left(\frac{1}{\sigma \sqrt{2 \pi}}\right)^{3} \exp \left(\frac{-\|\mathbf{x}\|_{2}^{2}}{2 \sigma^{2}}\right), 10^{-8}\right\}, & \ell=1 \\ 0, & \ell>1\end{cases}
$$

on a periodic domain of size $[-1,1]^{3}$ with $\sigma=0.03$ for the Gaussian initial condition. Figure 1 shows $\langle f\rangle$ in the $z=0$ plane for the two initial conditions.
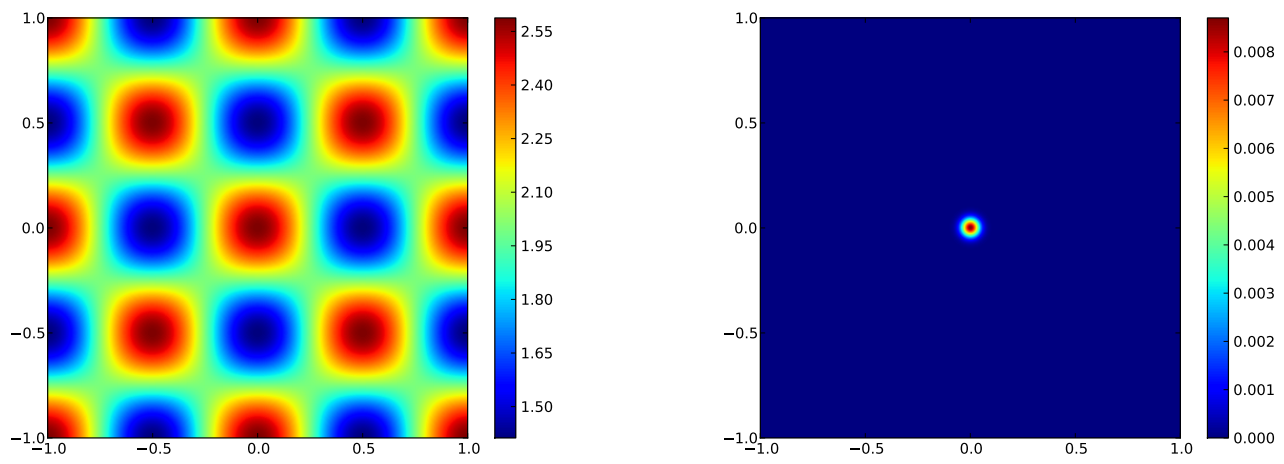

Figure 1: Left: smooth initial condition (14). Right: Gaussian initial condition (15). Figure shows $\langle f\rangle$ in the $z=0$ plane.

\section{Computational improvements for $\mathbf{M}_{N}$}

In this section, three computational optimizations for the $\mathrm{M}_{N}$ method will be detailed with results showing the efficacy for each. The results were computed using one node of the supercomputer Titan. Each node consists of a 16-core $2.2 \mathrm{GHz}$ AMD Opteron 6274 processor and an NVIDIA Tesla K20 GPU.

Before discussing the computational optimizations, it is necessary to know which functions comprise the bulk of the computation time. Table 1 contains timings for each of the four functions in Algorithm 2 executed on one compute node. From the table, it is clear that the bulk of the computing time is taken by the functions min and flux_mn. We also present a profile of the $\mathrm{P}_{N}$ method for comparison in Table 2. It is worth noting that the comm and euler functions have almost identical execution times when comparing $\mathrm{P}_{N}$ to $\mathrm{M}_{N}$. This is expected since these functions are identical in both cases. 


\begin{tabular}{lrr}
\hline Function & \multicolumn{1}{c}{ Time } & \% Time \\
\hline comm & $0.139 \mathrm{~s}$ & $0.11 \%$ \\
min & $99.764 \mathrm{~s}$ & $80.00 \%$ \\
flux_mn & $24.683 \mathrm{~s}$ & $19.79 \%$ \\
euler & $0.036 \mathrm{~s}$ & $0.03 \%$ \\
Sum & $124.622 \mathrm{~s}$ & $99.94 \%$ \\
\hline
\end{tabular}

(a) $\mathrm{M}_{3}$ method with $n_{g}=12$. Total wall clock time was $124.702 \mathrm{~s}$.

\begin{tabular}{lrr}
\hline Function & \multicolumn{1}{c}{ Time } & \% Time \\
\hline comm & $0.435 \mathrm{~s}$ & $0.02 \%$ \\
min & $2083.208 \mathrm{~s}$ & $87.71 \%$ \\
flux_mn & $290.745 \mathrm{~s}$ & $12.24 \%$ \\
euler & $0.235 \mathrm{~s}$ & $0.01 \%$ \\
Sum & $2374.623 \mathrm{~s}$ & $99.98 \%$ \\
\hline
\end{tabular}

(b) $\mathrm{M}_{7}$ method with $n_{g}=20$. Total wall clock time was 2375.041s.

Table 1: Profile of OpenMP code for Algorithm 2 with no optimizations. Parameters: smooth initial condition, $40^{3}$ spatial cells, 20 time steps taken.

\begin{tabular}{lrr}
\hline Function & Time & \% Time \\
\hline comm & $0.133 \mathrm{~s}$ & $7.60 \%$ \\
flux_pn & $1.559 \mathrm{~s}$ & $89.03 \%$ \\
euler & $0.028 \mathrm{~s}$ & $1.60 \%$ \\
Sum & $1.720 \mathrm{~s}$ & $98.23 \%$ \\
\hline
\end{tabular}

(a) $\mathrm{P}_{3}$ method. Total wall clock time was $1.751 \mathrm{~s}$.

\begin{tabular}{lrr}
\hline Function & Time & \% Time \\
\hline comm & $0.428 \mathrm{~s}$ & $2.61 \%$ \\
flux_pn & $15.535 \mathrm{~s}$ & $94.60 \%$ \\
euler & $0.225 \mathrm{~s}$ & $1.37 \%$ \\
Sum & $16.188 \mathrm{~s}$ & $98.58 \%$ \\
\hline
\end{tabular}

(b) $\mathrm{P}_{7}$ method. Total wall clock time was $16.422 \mathrm{~s}$.

Table 2: Profile of OpenMP code for Algorithm 3. Parameters: smooth initial condition, $40^{3}$ spatial cells, 20 time steps taken.

Remark 1. For clarity in the remainder of the paper, we will use the term "optimization" to refer to computational optimizations and the term "minimization" to refer to the minimization from Equation (6).

\subsection{GPU acceleration of the flux calculation}

Before describing the algorithm for GPU acceleration of flux_mn, we describe the CPU implementation of both flux_pn in Algorithm 4 and flux_mn in Algorithm 5 for comparison. To leading order, for a single spatial cell, the calculation of $\boldsymbol{\delta}_{i j k}$ for $\mathrm{P}_{N}$ takes $6 M^{2}$ multiplications to compute and for $\mathrm{M}_{N}$ takes $3 Q M$ multiplications to compute. Since $Q>>M$ in general, the calculation for $\mathrm{P}_{N}$ is much less costly than the calculation for $\mathrm{M}_{N}$. 

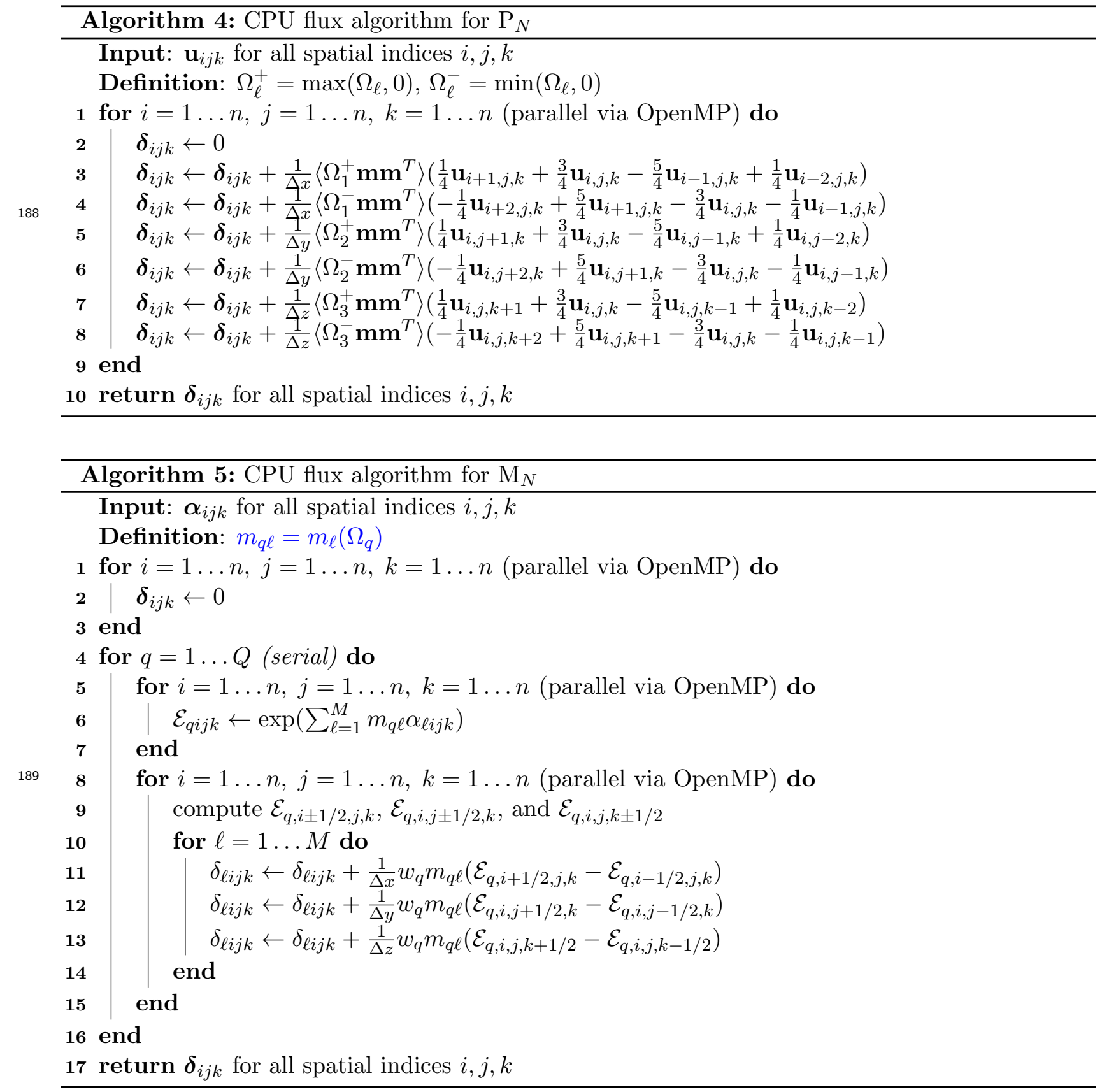

Two important implementation notes are in order. First, the integrals $\left\langle\Omega_{\ell}^{ \pm} \mathbf{m m}^{T}\right\rangle$ for $\ell=$ 1,2,3 from Algorithm 4 can be precomputed once at the beginning of the program. Second, for Algorithm 5, the outer loop in quadrature index starting at line 4 is done serially to save space in memory. This enables the variable $\mathcal{E}_{q i j k}$ to be held as only one floating point number per spatial cell instead of $Q$ floating point numbers per spatial cell. For a moment method, only $O\left(M n^{3}\right)$ bytes of memory should be required for the code, but if $\mathcal{E}_{q i j k}$ required $Q$ floating point numbers per spatial cell, the memory requirement would jump to $O\left(Q n^{3}\right)$ bytes. This is undesirable since $Q$ is generally much larger than $M$. For our simulations, there was plenty of work to do in parallel for the loops in spatial cells $i, j, k$, that we still got near perfect speedup, and thus parallelization in $q$ was not necessary. 
GPU acceleration of flux mn is given in Algorithm 6. The bold GPU function in the algorithm refers to a function computed on the GPU. Hence in this implementation, the algorithm calls three separate GPU functions. (Data copies between CPU and GPU were negligible compared to the computation times on the GPU.)

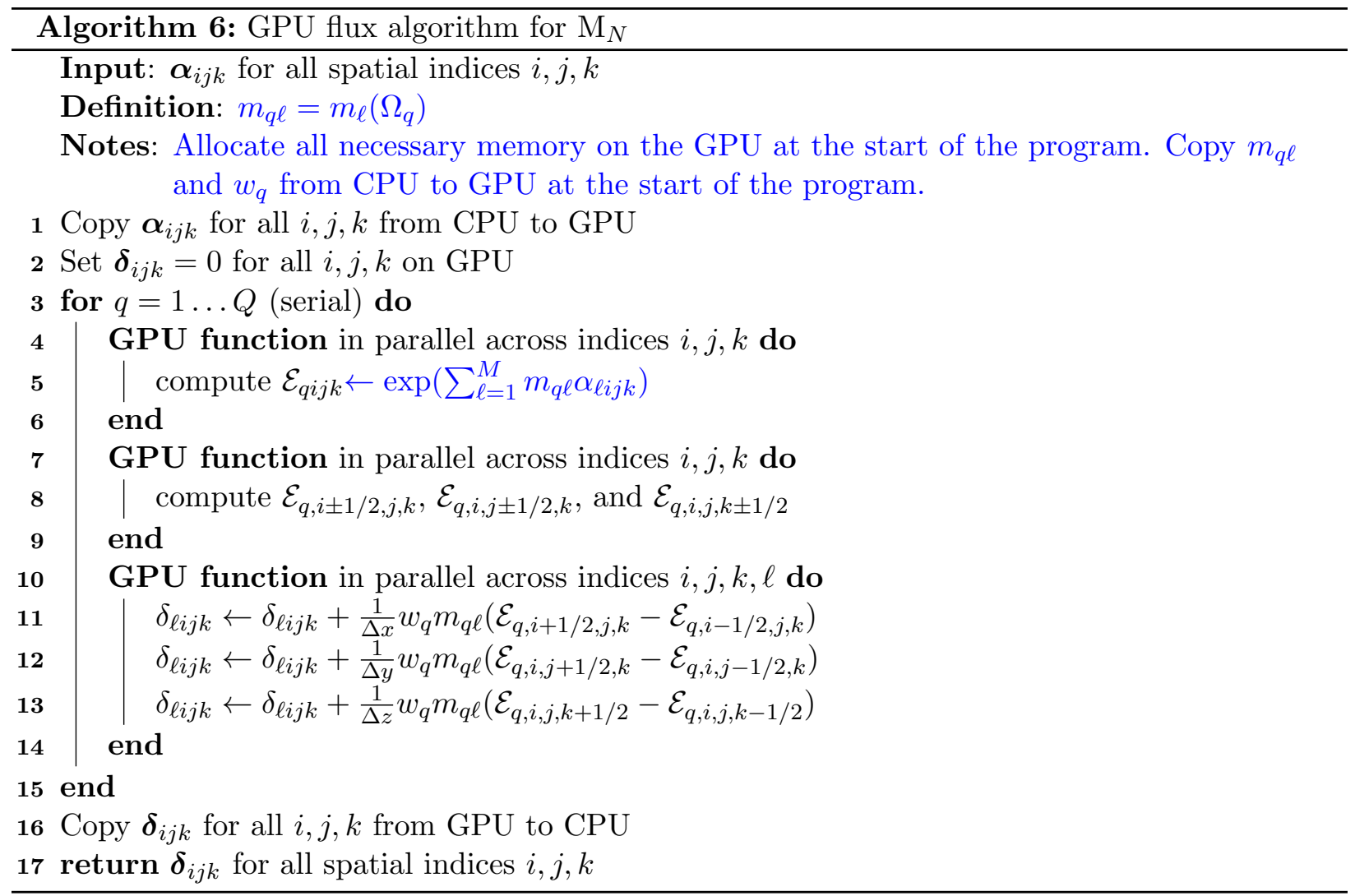

Acceleration results. As shown in Table 3, GPU acceleration of the flux calculation is significant. Results are given for the smooth initial condition with $40^{3}$ spatial cells and 20 time steps. The times given only include time elapsed inside the flux_mn function. The GPU acceleration ranges from $3 \mathrm{x}$ to almost $8 \mathrm{x}$ faster depending on the number of moments. The quadrature size does not seem to have much of an impact on the rate of GPU acceleration given our implementation.

\begin{tabular}{rrrrr}
\hline$N$ & $n_{g}$ & OpenMP & CUDA & Speedup \\
\hline 3 & 12 & $24.537 \mathrm{~s}$ & $8.169 \mathrm{~s}$ & $3.00 \mathrm{x}$ \\
3 & 20 & $68.289 \mathrm{~s}$ & $22.109 \mathrm{~s}$ & $3.09 \mathrm{x}$ \\
9 & 20 & $438.341 \mathrm{~s}$ & $57.113 \mathrm{~s}$ & $7.67 \mathrm{x}$ \\
9 & 28 & $858.825 \mathrm{~s}$ & $110.182 \mathrm{~s}$ & $7.79 \mathrm{x}$ \\
\hline
\end{tabular}

Table 3: Timings for the function flux_mn, using OpenMP vs CUDA.

\subsection{Evaluating the Hessian using real Gaunt coefficients}

To make the Hessian evaluation more efficient, we have employed an optimization using real Gaunt coefficients [22]. In a one-dimensional spatial setting, $\mathbf{m}$ reduces to a basis of $N+1$ Legendre 


\footnotetext{
${ }^{7}$ If $m_{\ell_{1}}$ has degree $d_{1}$ and $m_{\ell_{2}}$ has degree $d_{2}$, then the index $\ell_{3}$ in the sum need only go from 1 to $\left(d_{1}+d_{2}+1\right)^{2}$. To have one compact notation, we set $\hat{M}$ to the maximum possible value which occurs for degree $N$ spherical harmonics.
}

polynomials. It was shown in [25] that for this case, the Hessian can be iteratively constructed using only the first $N$ moments by exploiting integration by parts. The same technique can be used in a Cartesian product of closed intervals (i.e. a closed rectangular prism) and a tensor product basis. However, when using spherical harmonics on the unit sphere, a similar scheme does not appear to be possible.

The entries of the Hessian matrix $H$ from Algorithm 1 are given by

$$
H_{\ell_{1} \ell_{2}}=\sum_{q=1}^{Q} m_{\ell_{1} q} m_{\ell_{2} q} T_{q},
$$

where $m_{\ell q}=m_{\ell}\left(\Omega_{q}\right), \mathbf{m}_{q}=\mathbf{m}\left(\Omega_{q}\right)=\left(m_{1 q}, \ldots, m_{M q}\right)$, and $T_{q}(\boldsymbol{\alpha})=w_{q} \exp \left(\boldsymbol{\alpha}^{T} \mathbf{m}_{q}\right)$. For fixed $\boldsymbol{\alpha}$, $T_{q}(\boldsymbol{\alpha})$ needs to be computed only once. Hence, evaluation of all the entries in $H(\boldsymbol{\alpha})$ using (16) requires $O\left(Q M^{2}\right)$ flops (see Table 4 ).

The evaluation of $H$ using Gaunt coefficients relies on the fact that the product of any two spherical harmonics is given by [22]

$$
m_{\ell_{1}} m_{\ell_{2}}=\sum_{\ell_{3}=1}^{\hat{M}} \beta_{\ell_{1} \ell_{2} \ell_{3}} \hat{m}_{\ell_{3}} .
$$

Here $\beta_{\ell_{1} \ell_{2} \ell_{3}}$ are the real Gaunt coefficients, $\hat{\mathbf{m}}$ is a vector of real spherical harmonics of degree up to and including $2 N$, and $\hat{M}=(2 N+1)^{2} \cdot{ }^{7}$ Combining (16) and (17) gives

$$
H_{\ell_{1} \ell_{2}}=\sum_{\ell_{3}=1}^{\hat{M}} \beta_{\ell_{1} \ell_{2} \ell_{3}} \sum_{q=1}^{Q} \hat{m}_{\ell_{3} q} T_{q} .
$$

One can further optimize the calculation (18) using the fact that $\beta$ is sparse. As shown in Appendix C, for $\ell_{1}, \ell_{2}$ fixed, $\beta_{\ell_{1} \ell_{2} \ell_{3}}$ has at most $\sqrt{\hat{M}}=2 N+1$ nonzeros. To store $\beta$ sparsely, we treat $\beta$ as a matrix with the indices $\ell_{1}, \ell_{2}$ giving the row index and $\ell_{3}$ giving the column index. Then we store $\beta$ in the commonly used "compressed sparse row" (CSR) format for sparse matrices [42, p. 90].

Table 4 gives the floating point operation counts for computing the Hessian using (16) and using (18) with both full and sparse formats. For the cross-product quadrature (cf. Section 2.2), typically $Q \gg M$, and the use of the full/sparse Gaunt coefficients speeds up computations considerably. In particular, evaluating the flux matrix $\langle\Omega \mathbf{m} \mathcal{E}(\mathbf{u})\rangle$ exactly for $\mathcal{E}(\mathbf{u}) \in \operatorname{span}\left\{m_{1}, \ldots, m_{M}\right\}$ requires $Q=2(N+1)^{2} \approx 2 M \gg \sqrt{\hat{M}}$. Since $\mathcal{E}(\mathbf{u}) \notin \operatorname{span}\left\{m_{1}, \ldots, m_{M}\right\}$ in general, it is typical to have $Q>2(N+1)^{2}$. As a rule of thumb, we also use an even value of $n_{g}$ (see quadrature section of Section 2.2) for symmetry concerns. We note that although the sparse Gaunt optimization always uses many fewer flops to compute the Hessian, the sparsity requires indexing into memory in a nonconsecutive way. Therefore, computational speedup is not always as large as the flop ratio. 


\begin{tabular}{llll}
\hline & No Gaunt & Full Gaunt & Sparse Gaunt \\
\hline Order & $O\left(Q M^{2}\right)$ & $O\left(\hat{M} M^{2}\right)$ & $O\left(\sqrt{\hat{M}} M^{2}\right)$ \\
Sums & $Q M+Q M^{2}$ & $Q M+Q \hat{M}+M^{2} \hat{M}$ & $Q M+Q \hat{M}+M^{2} \sqrt{\hat{M}}$ \\
Mult & $Q+Q M+2 Q M^{2}$ & $Q+Q M+Q \hat{M}+M^{2} \hat{M}$ & $Q+Q M+Q \hat{M}+M^{2} \sqrt{\hat{M}}$ \\
$\operatorname{Exp}$ & $Q$ & $Q$ & $Q$ \\
\hline
\end{tabular}

Table 4: Floating point operation counts for the three methods of computing the Hessian $H$. "No Gaunt" means $H$ is computed by (16); "Full Gaunt" means $H$ is computed by (18) without using the sparsity of $\beta$; "Sparse Gaunt" means $H$ is computed by (18) using the sparsity of $\beta$.

Gaunt coefficients results. In Table 5, results are shown for the various Gaunt coefficients optimizations with both the CPU and GPU implementations. The timing results are for 20 time steps with the smooth initial condition on a $20^{3}$ domain with a batch size of 300 for the GPU implementation. (Batching on the GPU is described in Section 3.3.) The times given only include time elapsed inside the min function. For a smaller number of moments, there is not much difference in computation time between the full and sparse Gaunt optimization. However, as the number of moments increases, the benefit of the sparse Gaunt optimization increases. This is true for both the CPU and GPU implementations. The GPU version always outperforms the CPU, particularly with a larger number of quadrature points.

\begin{tabular}{rrrrrrrr}
\hline & \multicolumn{3}{c}{ No Gaunt } & \multicolumn{2}{c}{ Full Gaunt } & \multicolumn{2}{c}{ Sparse Gaunt } \\
$N$ & $n_{g}$ & \multicolumn{1}{c}{ CPU } & \multicolumn{1}{c}{ GPU } & CPU & GPU & CPU & GPU \\
\hline 3 & 12 & $12.4 \mathrm{~s}$ & $8.6 \mathrm{~s}$ & $8.7 \mathrm{~s}$ & $7.3 \mathrm{~s}$ & $8.1 \mathrm{~s}$ & $7.1 \mathrm{~s}$ \\
3 & 20 & $29.9 \mathrm{~s}$ & $12.2 \mathrm{~s}$ & $22.7 \mathrm{~s}$ & $7.9 \mathrm{~s}$ & $20.9 \mathrm{~s}$ & $7.8 \mathrm{~s}$ \\
9 & 20 & $576.5 \mathrm{~s}$ & $294.0 \mathrm{~s}$ & $491.3 \mathrm{~s}$ & $138.8 \mathrm{~s}$ & $98.2 \mathrm{~s}$ & $71.5 \mathrm{~s}$ \\
9 & 28 & $1107.0 \mathrm{~s}$ & $510.1 \mathrm{~s}$ & $580.3 \mathrm{~s}$ & $146.2 \mathrm{~s}$ & $167.8 \mathrm{~s}$ & $79.2 \mathrm{~s}$ \\
\hline
\end{tabular}

Table 5: CPU/GPU results for various Gaunt coefficients optimizations.

\subsection{GPU acceleration via batch Hessian computations}

There are several possible strategies for accelerating the minimization algorithm (Algorithm 1) with the GPU. One approach is to implement Algorithm 1 completely on the GPU. However, the performance of the algorithm is degraded in this case because the minimization algorithm as a whole is not easily vectorized. A second approach is to call the GPU every time a Hessian evaluation is required, since this is the most expensive part of the calculation. Such an implementation, however, results in frequent data transfers to and from the GPU. Thus, except for the case of large numbers of moments, there is not enough work for the GPU per function call to achieve good performance. An approach that yields good performance gains for both small and large moment orders is to batch solve several Hessian calculations, associated to different moments $\mathbf{u}_{i j k}$ of the mesh, at once on the GPU.

To implement the batching strategy, we separate each Newton iteration into the evaluation of the Hessian and the remainder of the iteration. We assemble a batch of independent minimization problems - possibly in different iterations of the algorithm - that all require a Hessian evaluation. The entire batch of Hessians is evaluated in parallel on the GPU and then moved to the CPU to 
complete the rest of each iteration. This process is detailed in Algorithm 7 and depicted in Figure 2.
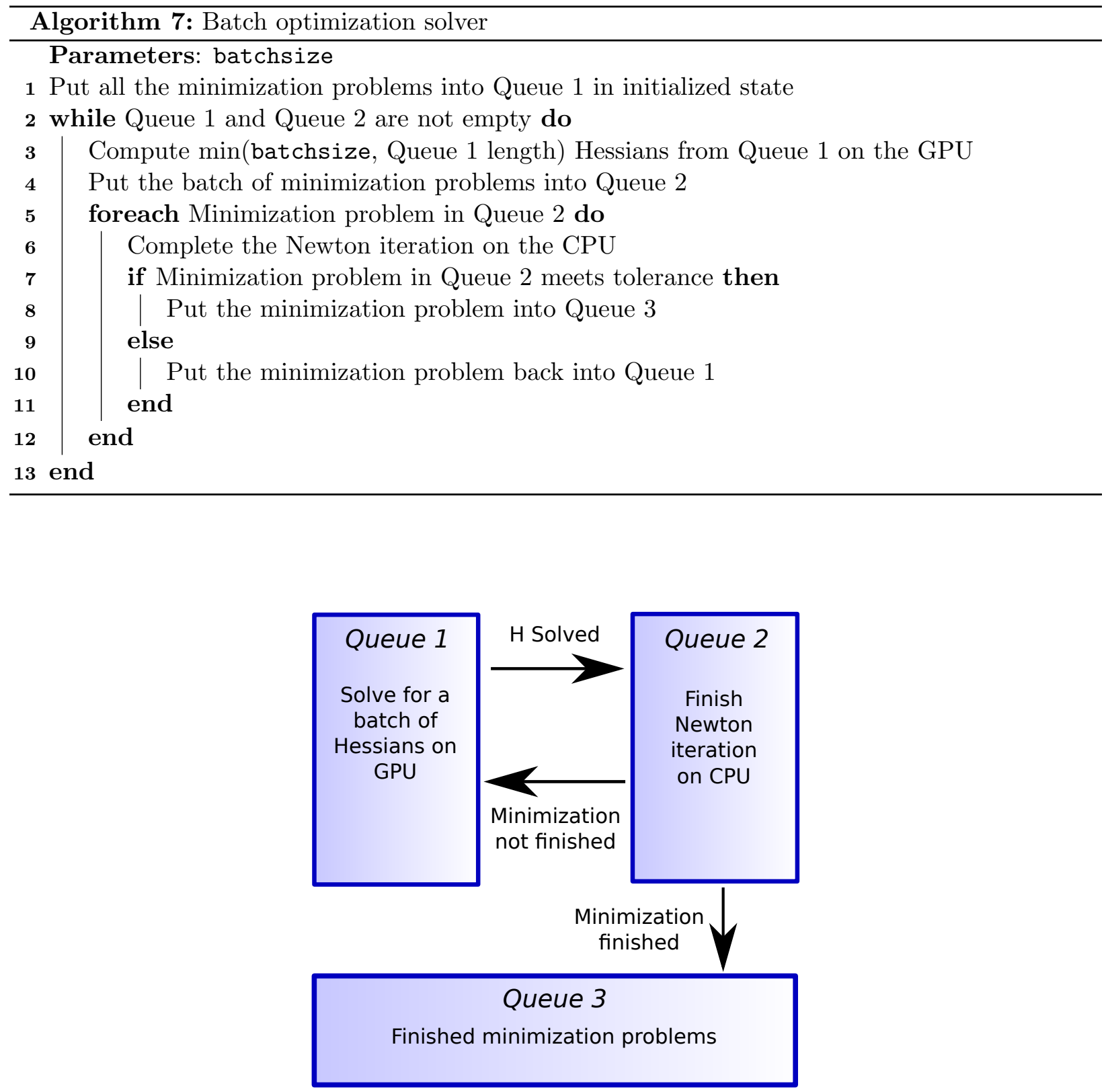

Figure 2: Figure describing the batch minimization algorithm with GPU acceleration.

Batch Hessian results. The batch size for the Hessian computations must be chosen large enough to give the GPU enough work to do. In Table 6, timing results are given for different batch sizes. The times given only include time elapsed inside the min function. Results are given for the smooth initial condition with $40^{3}$ spatial cells and 20 time steps while using the sparse Gaunt optimization. In general, the compute time is a decreasing function of batch size. 


\begin{tabular}{rrrrrrrrrr}
\hline$N$ & $n_{g}$ & \multicolumn{1}{c}{ CPU } & GPU-25 & GPU-50 & GPU-100 & GPU-200 & GPU-400 & GPU-800 & GPU-1600 \\
\hline 3 & 12 & $112.8 \mathrm{~s}$ & $122.1 \mathrm{~s}$ & $93.6 \mathrm{~s}$ & $79.4 \mathrm{~s}$ & $73.9 \mathrm{~s}$ & $69.9 \mathrm{~s}$ & $67.1 \mathrm{~s}$ & $65.7 \mathrm{~s}$ \\
3 & 20 & $238.0 \mathrm{~s}$ & $167.4 \mathrm{~s}$ & $118.0 \mathrm{~s}$ & $95.2 \mathrm{~s}$ & $82.3 \mathrm{~s}$ & $75.9 \mathrm{~s}$ & $72.1 \mathrm{~s}$ & $70.2 \mathrm{~s}$ \\
9 & 20 & $927.6 \mathrm{~s}$ & $999.3 \mathrm{~s}$ & $848.6 \mathrm{~s}$ & $755.1 \mathrm{~s}$ & $724.5 \mathrm{~s}$ & $714.7 \mathrm{~s}$ & $710.3 \mathrm{~s}$ & $708.3 \mathrm{~s}$ \\
9 & 28 & $1620.7 \mathrm{~s}$ & $1190.7 \mathrm{~s}$ & $941.3 \mathrm{~s}$ & $846.4 \mathrm{~s}$ & $797.4 \mathrm{~s}$ & $782.6 \mathrm{~s}$ & $778.5 \mathrm{~s}$ & $774.7 \mathrm{~s}$ \\
\hline
\end{tabular}

Table 6: Results using different batch sizes for calculating the Hessian on the GPU. GPU- $B$ represents the time elapsed using the GPU with a batch size of $B$.

\section{$4 \quad$ Statistics and scaling}

In this section, we present overall program results including (i) convergence results confirming second-order space-time accuracy; (ii) composite acceleration results from the combined improvements in Section 3; (iii) statistics on the number of iterations needed to solve the minimization problems; (iv) extensive timing data for a large scale $\mathrm{M}_{3}$ computation; and (v) weak scaling results for $\mathrm{P}_{3}$ and $\mathrm{M}_{3}$. All computations were performed on the supercomputer Titan.

Convergence. The convergence rate was tested using the smooth initial condition (14). The reference solution was computed using a spatial resolution of $n=320$, and the time step used for all calculations was $\Delta t=\frac{0.9}{6} \Delta x=0.15 \Delta x$ (see (13) and note $\Delta x=\Delta y=\Delta z$ ).

Table 7 shows $L^{2}$ spatial convergence data at time $t=0.3$. The table shows convergence data for moments of degree $d=0,1,2,3$. Since there are actually $2 d+1$ moments of degree $d$, we evaluate

$$
e_{d}=\left(\sum_{r=-d}^{d}\left\|u_{d, 320}^{r}-u_{d, n}^{r}\right\|_{2}^{2}\right)^{1 / 2},
$$

where $u_{d, n}^{r}$ is the degree $d$, order $r$ moment of the numerical solution, computed on the spatial mesh of size $n$. The results in Table 7 confirm second-order temporal and spatial convergence.

\begin{tabular}{ccccccccc}
\hline$n$ & $e_{0}$ & Conv Rate & $e_{1}$ & Conv Rate & $e_{2}$ & Conv Rate & $e_{3}$ & Conv Rate \\
\hline 10 & $1.22 \mathrm{e}-4$ & - & $4.28 \mathrm{e}-4$ & - & $5.46 \mathrm{e}-4$ & - & $4.70 \mathrm{e}-4$ & - \\
20 & $3.79 \mathrm{e}-5$ & 1.69 & $1.10 \mathrm{e}-4$ & 1.97 & $1.29 \mathrm{e}-4$ & 2.08 & $9.23 \mathrm{e}-5$ & 2.35 \\
40 & $1.38 \mathrm{e}-5$ & 1.45 & $2.39 \mathrm{e}-5$ & 2.20 & $2.86 \mathrm{e}-5$ & 2.17 & $2.53 \mathrm{e}-5$ & 1.87 \\
80 & $4.35 \mathrm{e}-6$ & 1.67 & $5.43 \mathrm{e}-6$ & 2.14 & $5.64 \mathrm{e}-6$ & 2.34 & $5.89 \mathrm{e}-6$ & 2.10 \\
160 & $7.88 \mathrm{e}-7$ & 2.47 & $9.38 \mathrm{e}-7$ & 2.53 & $1.04 \mathrm{e}-6$ & 2.44 & $1.22 \mathrm{e}-6$ & 2.27 \\
\hline
\end{tabular}

Table 7: Convergence data for $\mathrm{M}_{3}$ and smooth initial condition (14).

Algorithm acceleration. We next explore the combined improvement of the optimizations discussed in Section 3. We use both the smooth (14) and Gaussian (15) initial conditions with a $20^{3}$ spatial mesh and a batch size of 300 for the Hessian calculations. The results are given in Table 8. The overall speedups for both initial conditions increase from $1.8 \mathrm{x}$ to $13 \mathrm{x}$, depending on the number of moments and the number of quadrature points. As these increase, the optimizations become more effective. 


\begin{tabular}{ccrrrr}
\hline IC & $N$ & $n_{g}$ & \multicolumn{1}{c}{ Before } & \multicolumn{1}{c}{ After } & Speedup \\
\hline Smooth & 3 & 12 & $15.417 \mathrm{~s}$ & $8.369 \mathrm{~s}$ & $1.84 \mathrm{x}$ \\
Smooth & 3 & 20 & $38.685 \mathrm{~s}$ & $11.272 \mathrm{~s}$ & $3.43 \mathrm{x}$ \\
Smooth & 9 & 20 & $598.694 \mathrm{~s}$ & $86.116 \mathrm{~s}$ & $6.95 \mathrm{x}$ \\
Smooth & 9 & 28 & $1265.605 \mathrm{~s}$ & $97.635 \mathrm{~s}$ & $12.96 \mathrm{x}$ \\
Gaussian & 3 & 12 & $9.146 \mathrm{~s}$ & $4.932 \mathrm{~s}$ & $1.85 \mathrm{x}$ \\
Gaussian & 3 & 20 & $23.199 \mathrm{~s}$ & $7.631 \mathrm{~s}$ & $3.04 \mathrm{x}$ \\
Gaussian & 9 & 20 & $354.984 \mathrm{~s}$ & $40.697 \mathrm{~s}$ & $8.72 \mathrm{x}$ \\
Gaussian & 9 & 28 & $692.014 \mathrm{~s}$ & $50.728 \mathrm{~s}$ & $13.64 \mathrm{x}$ \\
\hline
\end{tabular}

Table 8: Overall improvement in time to solution for $\mathrm{M}_{N}$ simulations with smooth (14) and Gaussian (15) initial conditions.

The first row in Table 8 can be predicted given previous data in the paper. In Table 1, for $\mathrm{M}_{3}$ with $n_{g}=12$, the function min takes approximately $80 \%$ of the calculation time and flux_mn takes approximately $20 \%$ of the time. From Table 3, we expect a speedup of 3x for flux mn, and from Table 5 , we expect a speedup of $12.4 / 7.1=1.75 \mathrm{x}$ for $\mathrm{min}$. Therefore, the estimated total speedup is $\frac{100}{80 / 1.75+20 / 3}$ or $1.91 \mathrm{x}$, which is quite close to the $1.84 \mathrm{x}$ speedup actually observed. The speedup results in other rows could theoretically be determined in the same manner, but an initial profile was not created for those cases.

Minimization iterations. The function min is the only function that may vary significantly in time to completion between different spatial cells. This happens because the number of Newton iterations needed for the algorithm to converge changes from cell to cell. To see this disparity, we plot in Figure 3 the number of iterations taken by min to converge for each spatial cell on the X-axis. A spatial mesh with $100^{3}$ cells is used. For the smooth initial condition (14), 25 time steps were taken, and for the Gaussian initial condition (15), 167 time steps were taken. For the smooth initial condition, the first time step requires more iterations than any other time step because of the anisotropy of the initial condition. After this, the algorithm uses the previous data in the min function and only 1 or 2 minimization iterations are required for convergence. For the Gaussian initial condition, the problem is always difficult to solve on the expanding wave front, requiring in some cases more than 20 iterations for convergence of the minimization algorithm. (Note: there are twice as many Euler steps shown in Figure 3 as there are time steps since the Heun method takes two Euler steps.) 

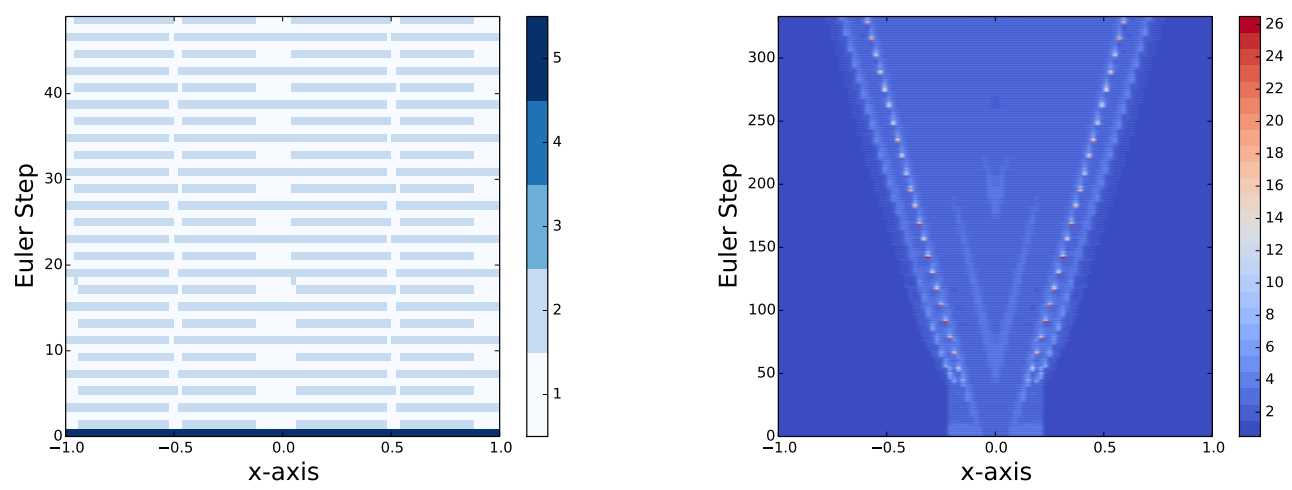

Figure 3: Plot of minimization iterations required for convergence for spatial cells on the $\mathrm{x}$-axis for each Euler step. Left: smooth initial condition (14). Notice the first Euler step requires 5 minimization iterations to converge. Right: Gaussian initial condition (15). Notice the large number of minimization iterations taken on the expanding wave front.

$\mathbf{M}_{3}$ statistics at scale. The variation in time taken by the min function for different spatial cells can cause load balancing problems for the $\mathrm{M}_{N}$ method that do not occur for the $\mathrm{P}_{N}$ method. To quantify this effect, we present timing data using the smooth initial condition (14) and a large parallel calculation: $26^{3}$ nodes with $100^{3}$ spatial cells per node. We present the elapsed time (time) for each of the four functions (comm, min, flux_mn, and euler) at each compute node (node) and each time step (step). The data has the form:

$$
\text { time }=\text { function(node, step). }
$$

When the stage of the time integrator from Algorithm 2 is important, these functions are separated into pairs: comm1/comm2, $\min 1 / \min 2, \mathrm{flux} 1 / \mathrm{flux} 2$, and euler1/euler2.

In Table 9, we aggregate the timing data, first summing the elapsed time for each function across time steps and then taking the mean and standard deviation of these elapsed times across nodes. We also present a normalized standard deviation calculated as standard deviation divided by mean.

Of particular importance is that the time for comm is not just the raw communication time; rather, it also includes the time spent waiting for data to be received. This is why the time for comm for $\mathrm{M}_{3}$ is so much larger than for $\mathrm{P}_{3}$. The standard deviation in time to compute a solution for $\mathrm{M}_{3}$ is larger than for $\mathrm{P}_{3}$, which causes more waiting time for receipt of data in certain nodes. A more detailed view of the timing data for $\mathrm{M}_{3}$ with $n=100$ is given in Figure 4, which shows the mean node timing of each function vs time step. 


\begin{tabular}{rrrrr}
\hline & \multicolumn{1}{c}{$n$} & Mean & Std & NStd \\
\hline $\mathrm{P}_{3}$ & 25 & $0.66 \mathrm{~s}$ & $0.22 \mathrm{~s}$ & 0.328 \\
$\mathrm{P}_{3}$ & 50 & $2.82 \mathrm{~s}$ & $0.83 \mathrm{~s}$ & 0.294 \\
$\mathrm{P}_{3}$ & 100 & $15.50 \mathrm{~s}$ & $7.29 \mathrm{~s}$ & 0.470 \\
$\mathrm{M}_{3}$ & 25 & $2.60 \mathrm{~s}$ & $1.83 \mathrm{~s}$ & 0.704 \\
$\mathrm{M}_{3}$ & 50 & $13.10 \mathrm{~s}$ & $12.00 \mathrm{~s}$ & 0.916 \\
$\mathrm{M}_{3}$ & 100 & $100.87 \mathrm{~s}$ & $88.49 \mathrm{~s}$ & 0.877 \\
\hline
\end{tabular}

(a) comm timings

\begin{tabular}{rrrcc}
\hline & \multicolumn{1}{c}{$n$} & Mean & Std & NStd \\
\hline $\mathrm{P}_{3}$ & 25 & $0.97 \mathrm{~s}$ & $0.15 \mathrm{~s}$ & 0.156 \\
$\mathrm{P}_{3}$ & 50 & $6.78 \mathrm{~s}$ & $0.87 \mathrm{~s}$ & 0.128 \\
$\mathrm{P}_{3}$ & 100 & $52.97 \mathrm{~s}$ & $7.37 \mathrm{~s}$ & 0.139 \\
$\mathrm{M}_{3}$ & 25 & $7.97 \mathrm{~s}$ & $0.11 \mathrm{~s}$ & 0.014 \\
$\mathrm{M}_{3}$ & 50 & $30.74 \mathrm{~s}$ & $0.31 \mathrm{~s}$ & 0.010 \\
$\mathrm{M}_{3}$ & 100 & $175.03 \mathrm{~s}$ & $0.58 \mathrm{~s}$ & 0.003 \\
\hline
\end{tabular}

(c) flux timings

\begin{tabular}{rrccc}
\hline & \multicolumn{1}{c}{$n$} & Mean & Std & NStd \\
\hline $\mathrm{P}_{3}$ & 25 & - & - & - \\
$\mathrm{P}_{3}$ & 50 & - & - & - \\
$\mathrm{P}_{3}$ & 100 & - & - & - \\
$\mathrm{M}_{3}$ & 25 & $36.07 \mathrm{~s}$ & $1.81 \mathrm{~s}$ & 0.050 \\
$\mathrm{M}_{3}$ & 50 & $228.43 \mathrm{~s}$ & $12.12 \mathrm{~s}$ & 0.053 \\
$\mathrm{M}_{3}$ & 100 & $1643.43 \mathrm{~s}$ & $89.54 \mathrm{~s}$ & 0.054 \\
\hline
\end{tabular}

(b) min timings

\begin{tabular}{rrccc}
\hline & $n$ & Mean & Std & NStd \\
\hline $\mathrm{P}_{3}$ & 25 & $0.02 \mathrm{~s}$ & $0.001 \mathrm{~s}$ & 0.066 \\
$\mathrm{P}_{3}$ & 50 & $0.20 \mathrm{~s}$ & $0.007 \mathrm{~s}$ & 0.036 \\
$\mathrm{P}_{3}$ & 100 & $2.45 \mathrm{~s}$ & $0.017 \mathrm{~s}$ & 0.007 \\
$\mathrm{M}_{3}$ & 25 & $0.07 \mathrm{~s}$ & $0.006 \mathrm{~s}$ & 0.093 \\
$\mathrm{M}_{3}$ & 50 & $0.46 \mathrm{~s}$ & $0.038 \mathrm{~s}$ & 0.084 \\
$\mathrm{M}_{3}$ & 100 & $3.84 \mathrm{~s}$ & $0.300 \mathrm{~s}$ & 0.078 \\
\hline
\end{tabular}

(d) euler timings

Table 9: $\mathrm{P}_{3} / \mathrm{M}_{3}$ timings on $26^{3}$ nodes. (Std: standard deviation; NStd: normalized standard deviation.)
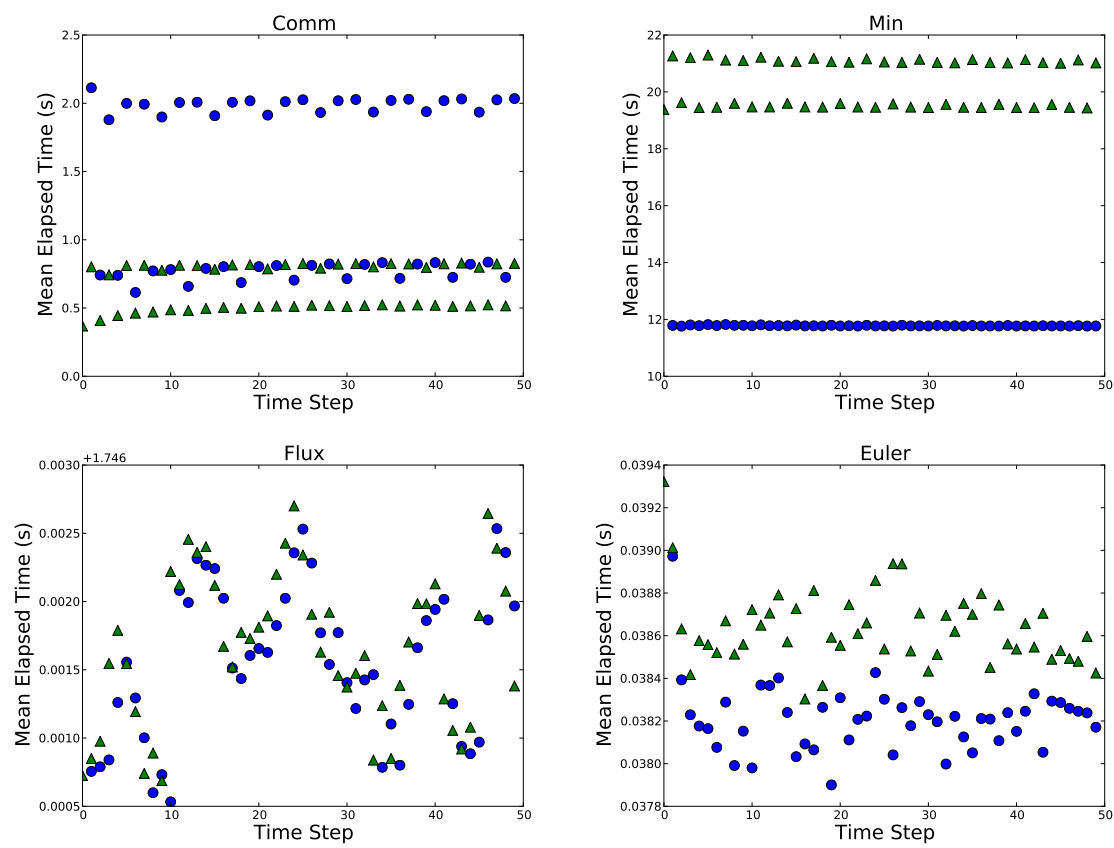

Figure 4: Mean node time is shown. Blue circles represent the first Euler step and green triangles represent the second Euler step.

The most striking feature of the data is that comm1 takes longer than comm2, while min2 takes 
longer than min1. We conjecture that there is a cause and effect relationship here in which the second min function takes longer to compute and more importantly has a higher standard deviation. This higher standard deviation then causes blocking of the communication to the first comm function. From Figure 3, we can see why min2 takes longer to compute than min1. The second Euler step requires on average 2 iterations to converge whereas the first Euler step requires on average only 1 iteration to converge. This may occur because the initial guess used for the minimization algorithm at the beginning of a Heun step uses the result of the previous Euler step ( $s=1$ from Algorithm 2), which is an approximation of the solution at the current time step.

Weak scaling results. The overall goal of $[2,3]$ and the work presented here is to make $\mathrm{M}_{N}$ more competitive with $\mathrm{P}_{N}$ in time to solution, specifically at scale. Therefore, we performed weak scaling tests for $\mathrm{P}_{3}$ and $\mathrm{M}_{3}$ on Titan using the smooth initial condition (14). We ran the scaling tests for $10^{3}, 25^{3}, 50^{3}$, and $100^{3}$ spatial cells per node from 1 node to $26^{3}=17576$ nodes. GPU acceleration was used to speed up the $\mathrm{M}_{3}$ calculations for both the flux_mn and min functions. A batch size of 300 was used for the Hessian computations, and sparse Gaunt coefficients were used. Figure 5 shows the weak scaling results, and Table 10 gives the difference in time to solution for a single node and for the largest scale computations. Table 11 compares the flop rates on one node for $\mathrm{P}_{3}$ and $\mathrm{M}_{3}$ flux computations. ${ }^{8}$
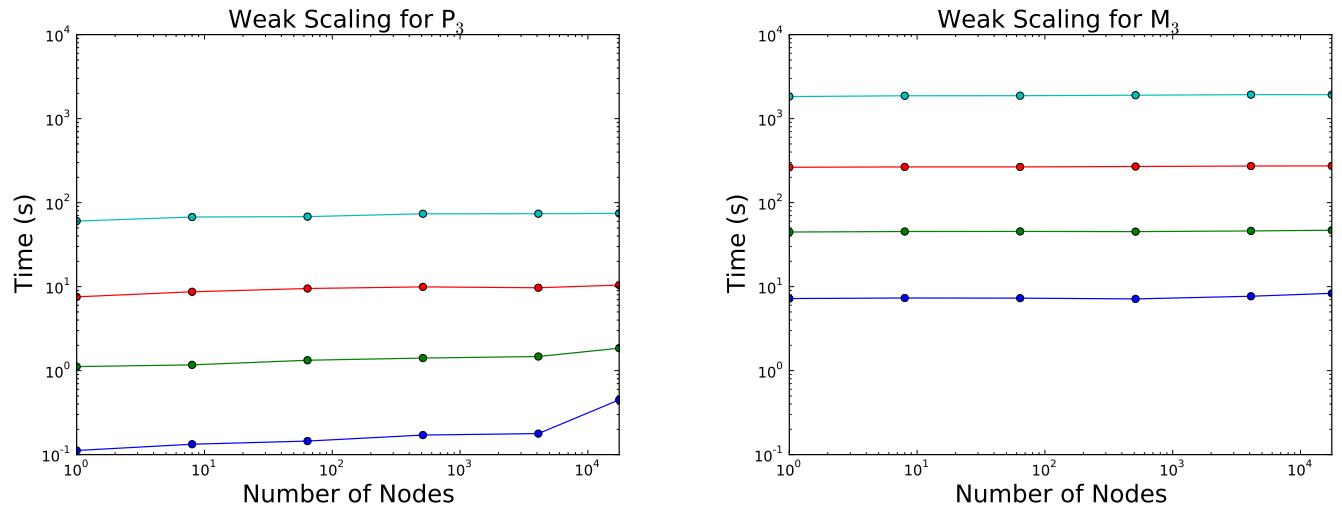

Figure 5: Weak scaling results (lines bottom to top in spatial cells per node: $10^{3}, 25^{3}, 50^{3}, 100^{3}$ ) for (left) $\mathrm{P}_{3}$, (right) $\mathrm{M}_{3}$.

\begin{tabular}{ccrcrrr}
\hline & \multicolumn{3}{c}{1 node } & \multicolumn{3}{c}{17576 nodes } \\
Size & \multicolumn{1}{c}{$\mathrm{P}_{3}$} & \multicolumn{1}{c}{$\mathrm{M}_{3}$} & $\mathrm{M}_{3} / \mathrm{P}_{3}$ & \multicolumn{1}{c}{$\mathrm{P}_{3}$} & \multicolumn{1}{c}{$\mathrm{M}_{3}$} & $\mathrm{M}_{3} / \mathrm{P}_{3}$ \\
\hline $10^{3}$ & $0.11 \mathrm{~s}$ & $7.21 \mathrm{~s}$ & $64.4 \mathrm{x}$ & $0.45 \mathrm{~s}$ & $8.32 \mathrm{~s}$ & $18.5 \mathrm{x}$ \\
$25^{3}$ & $1.12 \mathrm{~s}$ & $44.68 \mathrm{~s}$ & $40.0 \mathrm{x}$ & $1.85 \mathrm{~s}$ & $46.94 \mathrm{~s}$ & $25.4 \mathrm{x}$ \\
$50^{3}$ & $7.55 \mathrm{~s}$ & $263.45 \mathrm{~s}$ & $34.9 \mathrm{x}$ & $10.44 \mathrm{~s}$ & $273.57 \mathrm{~s}$ & $26.2 \mathrm{x}$ \\
$100^{3}$ & $60.31 \mathrm{~s}$ & $1831.37 \mathrm{~s}$ & $30.4 \mathrm{x}$ & $74.47 \mathrm{~s}$ & $1928.03 \mathrm{~s}$ & $25.9 \mathrm{x}$ \\
\hline
\end{tabular}

Table 10: Table comparing time to solution for 1 node and 17576 nodes. Size is the number of spatial cells per node.

\footnotetext{
${ }^{8}$ Flop rates are similar per node up to $26^{3}$ nodes.
} 


\begin{tabular}{rrr}
\hline \multicolumn{1}{c}{$n$} & $\mathrm{P}_{3}$ Flop Rate & \multicolumn{1}{c}{$\mathrm{M}_{3}$ Flop Rate } \\
\hline 10 & 6.32 GFLOPS & 2.00 GFLOPS \\
25 & 6.31 GFLOPS & 9.57 GFLOPS \\
50 & 7.46 GFLOPS & 21.83 GFLOPS \\
100 & 7.48 GFLOPS & 32.44 GFLOPS \\
\hline
\end{tabular}

Table 11: The flux_pn and flux_mn flop rates for one node. Note the flop rates never reach peak performance because the computations are memory bandwidth limited.

We observe that the $\mathrm{P}_{3}$ solution time does increase with the number of nodes, but only for a very small problem size $\left(10^{3}\right.$ cells per node) does the simulation become communication dominated. In this case, the $\mathrm{M}_{3}$ calculation becomes more competitive at scale, but the $\mathrm{P}_{3}$ simulation is still about 25 times faster. Thus for the same time to solution, the $P_{3}$ simulation with the current numerical method can afford a spatial resolution that is $2-3$ times finer than the corresponding $\mathrm{M}_{3}$ simulation at the largest scale. Depending on the problem at hand, $\mathrm{M}_{N}$ may be a better closure at any scale if Gibbs phenomena in the angular approximation strongly pollutes the $\mathrm{P}_{N}$ solution and, at the same, less spatial resolution can be tolerated. We also note the disparity in the flop rates between the $\mathrm{M}_{3}$ and $\mathrm{P}_{3}$ implementations due to the use of CUDA for $\mathrm{M}_{3}$ and OpenMP for $\mathrm{P}_{3}$. Clearly, there is room for improvement in the implementation of the $\mathrm{P}_{N}$ method if GPU acceleration is used to accelerate the flux_pn function.

\section{Conclusions and discussion}

Several optimizations were shown to accelerate the implementation of the entropy-based model $\mathrm{M}_{N}$. The use of Gaunt coefficients, in conjunction with GPU acceleration strategies, reduces the time to solution for a given test problem between $1.8 \mathrm{x}$ and $13 \mathrm{x}$. We also computed performance statistics for $\mathrm{M}_{N}$, and it was shown that $\mathrm{M}_{N}$ can cause some load balancing issues at scale. Finally, we compared weak scaling results of the $\mathrm{P}_{N}$ and $\mathrm{M}_{N}$ algorithms. For our test problems, the ratio of $\mathrm{M}_{N}$ to $\mathrm{P}_{N}$ in time to solution is reduced from $64 \mathrm{x}$ on 1 node to $18 \mathrm{x}$ on 17576 nodes for the smallest problem per node and from 30x on 1 node to 26x on 17576 nodes for the largest problem size per node.

We conjecture that the weak scaling results presented here provide a reasonable lower bound of what can be expected for the relative efficiency of the $\mathrm{M}_{N}$ calculation. One reason for this is the simplicity of the model. Indeed, most increases in model complexity, such as a more realistic collision operator, will require a similar increase in floating-point operations and memory resources for both $\mathrm{P}_{N}$ and $\mathrm{M}_{N}$ models, thereby decreasing the ratio in time to solution of $\mathrm{M}_{N}$ relative to $\mathrm{P}_{N}$. A second reason is the simplicity of the explicit time integrator. Indeed, for many applications, an implicit time integration scheme is desired to allow for large time steps. Such schemes involve global communication of data between all nodes, as opposed to the communication of halo data between adjacent nodes that is characteristic of explicit schemes. An implicit time integration scheme would require a linear global solve for the $\mathrm{P}_{N}$ equations, but a global nonlinear solve for the $\mathrm{M}_{N}$ equations. Thus the communication costs for $\mathrm{M}_{N}$ are likely to be higher. However, as both methods become increasingly communication-bound, the ratio of time to solution between them will continue to decrease. In addition, the use of iterative solvers means that one could, in many cases, solve the $\mathrm{M}_{N}$ optimization problem rather coarsely in earlier iterations.

In a larger context, the scaling results in Figure 5 and Table 10 give some insight into algorithm development for emerging architectures at extreme scales. It is generally expected [37] that these 
new systems will be increasingly limited - both in terms of speed and cost - by memory storage and data transfer. The Titan supercomputer is just the very beginning of this evolution. New systems will lend themselves to algorithms that are arithmetically intensive, i.e., where the number of floating point operations per unit of memory is large. Thus, in order to gain accuracy in a simulation, it is natural to ask whether, at some point, it is more efficient to move from a simple, linear model to a complicated, highly nonlinear model (where more floating point operations are required) instead of simply increasing the number of unknowns (where more memory is required). The choice between $\mathrm{P}_{N}$ and $\mathrm{M}_{N}$ exemplifies this choice, and the scaling results tell us two things. First, the data transfer capabilities of Titan are still good enough to give $\mathrm{P}_{N}$ a distinct advantage in time to solution, even for full-scale simulations. Second, however, one can clearly observe the slowdown caused by memory movement at the very highest scales, particularly when the memory per node is very low. While this amount of memory per node is unreasonably low, it is important to note that the ratio of memory size to number of floating point units will continue to decrease as the system size increases.

One important comparison between $\mathrm{M}_{N}$ and $\mathrm{P}_{N}$ that has not been done here is an analysis of efficiency that compares the efficiency of the two methods, both in terms of time and energy, for realistic physics problems. For a given problem, one should determine for each method what value of $N$ is needed to attain a prescribed level of accuracy. Based on the two values of $N$, one should calculate in each case the time to solution and the energy costs and then make comparisons. Such a project is currently work in progress.

\section{A Real spherical harmonics}

We used the real spherical harmonics as an orthonormal basis for $L^{2}\left(\mathbb{S}^{2}\right)$. For $d \geq 0$ and $-d \leq r \leq d$, the degree $d$ and order $r$ normalized, real spherical harmonic is given by

$$
R_{d}^{r}(\theta, \phi)= \begin{cases}\sqrt{2} N_{d}^{-r} P_{d}^{-r}(\cos \theta) \sin (r \phi), & r<0 \\ N_{d}^{0} P_{d}(\cos \theta), & r=0 \\ \sqrt{2} N_{d}^{r} P_{d}^{r}(\cos \theta) \cos (r \phi), & r>0\end{cases}
$$

where

$$
N_{d}^{r}=\sqrt{\frac{(2 d+1)}{4 \pi} \frac{(d-r) !}{(d+r) !}}
$$

$$
P_{d}^{r}(\mu)=(-1)^{r}\left(1-\mu^{2}\right)^{r / 2} \frac{d^{r}}{d \mu^{r}} P_{d}(\mu), \quad r>0 ;
$$

and $P_{d}$ is the degree $d$ Legendre polynomial, normalized so that $\int_{-1}^{1} P_{d}(\mu) d \mu=\frac{2}{2 d+1}$. The spherical harmonics are packed into $\mathbf{m}$ as

$$
\mathbf{m}=\left(R_{0}^{0}, R_{1}^{-1}, R_{1}^{0}, R_{1}^{1}, \ldots, R_{N}^{-N}, \ldots, R_{N}^{N}\right) .
$$

\section{B Solving for the real Gaunt coefficients}

To solve for the real Gaunt coefficients $\beta_{\ell_{1} \ell_{2} \ell_{3}}$, notice that (17) must be true for all points and in particular the quadrature points on the sphere described in Section 2.2. Therefore, for $q=1 \ldots Q$,

$$
m_{\ell_{1} q} m_{\ell_{2} q}=\sum_{\ell_{3}=1}^{\hat{M}} \beta_{\ell_{1} \ell_{2} \ell_{3}} \hat{m}_{\ell_{3} q}
$$


Taking $\ell_{1}$ and $\ell_{2}$ to be constant in the above equation, this is just an overdetermined linear system (assuming $Q>\hat{M})$. Denote the left hand side vector of (25) as $\mathbf{v}^{\left(\ell_{1} \ell_{2}\right)}$ (has length $Q$ ), the matrix given by $\hat{m}_{\ell_{3} q}$ as $A$ (has size $Q \times \hat{M}$ ), and the vector given by $\beta_{\ell_{1} \ell_{2} \ell_{3}}$ as $\mathbf{b}^{\left(\ell_{1} \ell_{2}\right)}$ (has length $\hat{M}$ ). Still with $\ell_{1}, \ell_{2}$ constant, this yields the linear system:

$$
\mathbf{v}^{\left(\ell_{1} \ell_{2}\right)}=A \mathbf{b}^{\left(\ell_{1} \ell_{2}\right)} .
$$

We solve the system as a least squares problem

$$
\min _{\mathbf{b}^{\left(\ell_{1} \ell_{2}\right)} \in \mathbb{R}^{\hat{M}}}\left\|\mathbf{v}^{\left(\ell_{1} \ell_{2}\right)}-A \mathbf{b}^{\left(\ell_{1} \ell_{2}\right)}\right\|_{2}
$$

using the method of computing a $\mathrm{QR}$ decomposition. Let $A=U R$, where $U$ is an orthogonal matrix and $R$ is an upper triangular matrix. Then

$$
\left\|\mathbf{v}^{\left(\ell_{1} \ell_{2}\right)}-A \mathbf{b}^{\left(\ell_{1} \ell_{2}\right)}\right\|_{2}=\left\|\mathbf{v}^{\left(\ell_{1} \ell_{2}\right)}-U R \mathbf{b}^{\left(\ell_{1} \ell_{2}\right)}\right\|_{2}=\left\|U^{T} \mathbf{v}^{\left(\ell_{1} \ell_{2}\right)}-R \mathbf{b}^{\left(\ell_{1} \ell_{2}\right)}\right\|_{2},
$$

where minimizing over the last expression is trivial. Since $A$ does not involve $\ell_{1}$ or $\ell_{2}$, only one QR decomposition is needed to solve the system over and over again for the different $\ell_{1}, \ell_{2}$. Also remember, the real Gaunt coefficients only need to be computed once at the beginning of the program.

\section{Sparsity result for the real Gaunt coefficients}

For the real spherical harmonics,

$$
m_{\ell_{1}} m_{\ell_{2}}=\sum_{\ell_{3}=1}^{\hat{M}} \beta_{\ell_{1} \ell_{2} \ell_{3}} \hat{m}_{\ell_{3}}
$$

where $\hat{M}=(2 N+1)^{2}, \mathbf{m}$ contains spherical harmonics of degree less than or equal to $N$, and $\hat{\mathbf{m}}$ contains spherical harmonics of degree less than or equal to $2 N$.

Proposition 1. For fixed $\ell_{1}, \ell_{2}$, the sum in (29) has no more than $2 N+1$ nonzero elements in it.

Proof. The real spherical harmonics of degree $d$ can be given in terms of the complex spherical harmonics via the unitary transformation [22]

$$
\left(\begin{array}{c}
R_{d}^{d} \\
\vdots \\
R_{d}^{1} \\
R_{d}^{0} \\
R_{d}^{-1} \\
\vdots \\
R_{d}^{-d}
\end{array}\right)=\frac{1}{\sqrt{2}}\left(\begin{array}{ccccccc}
1 & & & & & & 1 \\
& \ddots & & & & . \cdot & \\
& & 1 & & 1 & & \\
& & & \sqrt{2} & & & \\
& & i & & -i & & \\
& . \cdot & & & & \ddots & \\
i & & & & & & -i
\end{array}\right)\left(\begin{array}{c}
Y_{d}^{d} \\
\vdots \\
Y_{d}^{1} \\
Y_{d}^{0} \\
Y_{d}^{-1} \\
\vdots \\
Y_{d}^{-d}
\end{array}\right)
$$

where the complex spherical harmonics are defined as

$$
Y_{d}^{r}(\theta, \phi)=N_{d}^{|r|} P_{d}^{|r|}(\cos \theta) e^{i r \phi}
$$


with $N_{d}^{r}$ and $P_{d}^{r}$ defined in Appendix A. The transformation (30) and its inverse can be written as

$$
\begin{aligned}
& R_{d}^{r}=a_{r} Y_{d}^{r}+b_{r} Y_{d}^{-r} \\
& Y_{d}^{r}=\bar{a}_{r} R_{d}^{r}+\bar{b}_{-r} R_{d}^{-r}
\end{aligned}
$$

where

$$
a_{r}=\frac{1}{\sqrt{2}}\left\{\begin{array}{ll}
1, & r>0 \\
\frac{\sqrt{2}}{2}, & r=0 \\
-i, & r<0
\end{array} \quad \text { and } \quad b_{r}=\frac{1}{\sqrt{2}} \begin{cases}1, & r>0 \\
\frac{\sqrt{2}}{2}, & r=0 \\
i, & r<0\end{cases}\right.
$$

and $\bar{a}$ denotes the complex conjugate of $a$.

Now, multiplying two real spherical harmonics and expanding yields

$$
\begin{aligned}
R_{d_{1}}^{r_{1}} R_{d_{2}}^{r_{2}}= & \left(a_{r_{1}} Y_{d_{1}}^{r_{1}}+b_{r_{1}} Y_{d_{1}}^{-r_{1}}\right)\left(a_{r_{2}} Y_{d_{2}}^{r_{2}}+b_{r_{2}} Y_{d_{2}}^{-r_{2}}\right) \\
= & a_{r_{1}} a_{r_{2}} Y_{d_{1}}^{r_{1}} Y_{d_{2}}^{r_{2}}+a_{r_{1}} b_{r_{2}} Y_{d_{1}}^{r_{1}} Y_{d_{2}}^{-r_{2}} \\
& +b_{r_{1}} a_{r_{2}} Y_{d_{1}}^{-r_{1}} Y_{d_{2}}^{r_{2}}+b_{r_{1}} b_{r_{2}} Y_{d_{1}}^{-r_{1}} Y_{d_{2}}^{-r_{2}} .
\end{aligned}
$$

Each product of two complex spherical harmonics can be written as the following linear combination of complex spherical harmonics using (complex) Gaunt coefficients [41]

$$
Y_{d_{1}}^{r_{1}} Y_{d_{2}}^{r_{2}}=\sum_{\substack{d=0 \\ 2 \mid\left(d+d_{1}+d_{2}\right)}}^{d_{1}+d_{2}} G_{d, d_{1}, d_{2}}^{r_{1}, r_{2}} Y_{d}^{r_{1}+r_{2}}
$$

In the sum (36), we use the notation $2 \mid\left(d+d_{1}+d_{2}\right)$ to represent $d+d_{1}+d_{2} \equiv 0(\bmod 2)$. Notice how we only have to sum over very few complex spherical harmonics. Now, putting together equations (35) and (36) yields

$$
\begin{aligned}
R_{d_{1}}^{r_{1}} R_{d_{2}}^{r_{2}}=\sum_{\substack{d=0 \\
2 \mid\left(d+d_{1}+d_{2}\right)}}^{d_{1}+d_{2}} & \left(a_{r_{1}} a_{r_{2}} G_{d, d_{1}, d_{2}}^{r_{1}, r_{2}} Y_{d}^{r_{1}+r_{2}}\right. \\
& +a_{r_{1}} b_{r_{2}} G_{d, d_{1}, d_{2}}^{r_{1},-r_{2}} Y_{d}^{r_{1}-r_{2}} \\
& +b_{r_{1}} a_{r_{2}} G_{d, d_{1}, d_{2}}^{-r_{1}, r_{2}} Y_{d}^{-r_{1}+r_{2}} \\
& \left.+b_{r_{1}} b_{r_{2}} G_{d, d_{1}, d_{2}}^{-r_{1},-r_{2}} Y_{d}^{-r_{1}-r_{2}}\right) .
\end{aligned}
$$

Label the four parts of the summand from (37) as (I), (II), (III), and (IV). Then expanding the right side of equation (37) back into real spherical harmonics using (33) and using the fact

$$
G_{d, d_{1}, d_{2}}^{r_{1}, r_{2}}=G_{d, d_{1}, d_{2}}^{-r_{1},-r_{2}}
$$

yields

$$
\begin{aligned}
& (I): \quad G_{d, d_{1}, d_{2}}^{r_{1}, r_{2}}\left(a_{r_{1}} a_{r_{2}} \bar{a}_{r_{1}+r_{2}}+b_{r_{1}} b_{r_{2}} \bar{b}_{r_{1}+r_{2}}\right) R_{d}^{r_{1}+r_{2}} \\
& (I I): \quad G_{d, d_{1}, d_{2}}^{r_{1}, c_{2}}\left(a_{r_{1}} b_{r_{2}} \bar{a}_{r_{1}-r_{2}}+b_{r_{1}} a_{r_{2}} \bar{b}_{r_{1}-r_{2}}\right) R_{d}^{r_{1}-r_{2}} \\
& (I I I): G_{d, d_{1}, d_{2}}^{-r_{1}, r_{2}}\left(b_{r_{1}} a_{r_{2}} \bar{a}_{-r_{1}+r_{2}}+a_{r_{1}} b_{r_{2}} \bar{b}_{-r_{1}+r_{2}}\right) R_{d}^{-r_{1}+r_{2}} \\
& (I V): \quad G_{d, d_{1}, d_{2}}^{-r_{1},-r_{2}}\left(b_{r_{1}} b_{r_{2}} \bar{a}_{-r_{1}-r_{2}}+a_{r_{1}} a_{r_{2}} \bar{b}_{-r_{1}-r_{2}}\right) R_{d}^{-r_{1}-r_{2}} .
\end{aligned}
$$

The coefficients of $R$ in (I), (II), (III), and (IV) yield the real Gaunt coefficients except when $r_{1}+r_{2}=0$ or $r_{1}-r_{2}=0$. In these cases, either (I) $+(\mathrm{IV})$ or (II) $+(\mathrm{III})$ yields the real Gaunt coefficient. 
Comparing the expressions $a_{r_{1}} a_{r_{2}} \bar{a}_{r_{1}+r_{2}}$ and $b_{r_{1}} b_{r_{2}} \bar{a}_{-r_{1}-r_{2}}$ from (I) and (IV) respectively, if $r_{1}+r_{2} \neq 0$, then exactly one of these expressions is real and the other one is purely imaginary and nonzero. The same is true for $b_{r_{1}} b_{r_{2}} \bar{b}_{r_{1}+r_{2}}$ and $a_{r_{1}} a_{r_{2}} \bar{b}_{-r_{1}-r_{2}}$. Since the coefficients of $R_{d}^{r_{1}+r_{2}}$ and $R_{d}^{-r_{1}-r_{2}}$ must be real, only one of (I) and (IV) is nonzero. In the case $r_{1}+r_{2}=0, R_{d}^{r_{1}+r_{2}}=$ $R_{d}^{-r_{1}-r_{2}}=R_{d}^{0}$ and the coefficients are added to form the real Gaunt coefficient. This same parity occurs for (II) and (III). Hence, (I), (II), (III), and (IV) represent at most two nonzero real Gaunt coefficients.

The maximum possible number of nonzero real Gaunt coefficients occurs when $d_{1}=d_{2}=N$, which gives the permissible $\ell$ values in sum (37) of

$$
d=0,2, \ldots, 2 N .
$$

For $d=0$, the only possible value of $m$ is zero yielding at most 1 real Gaunt coefficient. For $d=2,4, \ldots, 2 N$, there are two possible nonzero real Gaunt coefficients, which yields a total of $2 N+1$ possible real Gaunt coefficients. This possibility is achieved by the product $R_{N}^{1} R_{N}^{1}$.

\section{Variables}

$n \quad$ - Number of spatial cells in a Cartesian direction ( $n^{3}$ total cells).

$N \quad$ - Maximal degree of moments.

$M \quad$ - Number of moments $\left(M=(N+1)^{2}\right)$.

$\hat{M} \quad$ - Number of moments needed in the computation of Gaunt coefficients $\left(\hat{M}=(2 N+1)^{2}\right)$.

$Q \quad$ - Number of quadrature points in angle.

$n_{g} \quad$ - Number of Gauss-Legendre quadrature points on $\Omega_{3}$-axis $\left(Q=2 n_{g}^{2}\right)$.

m - Vector of real spherical harmonics up to and including degree $N$.

$\hat{\mathbf{m}}$ - Vector of real spherical harmonics up to and including degree $2 N$.

$i, j, k$ - Indices for spatial coordinates.

$q \quad$ - Index for quadrature in angle.

$\ell \quad-$ Index for entry in a vector.

$d, r \quad$ - Degree and order indices respectively for spherical harmonics.

\section{Acknowledgments}

This research used resources of the Oak Ridge Leadership Computing Facility at the Oak Ridge National Laboratory, which is supported by the Office of Science of the U.S. Department of Energy under Contract No. DE-AC05-00OR22725.

This research is sponsored by the Office of Advanced Scientific Computing Research; U.S. Department of Energy. The work was performed at the Oak Ridge National Laboratory, which is managed by UT-Battelle, LLC under Contract No. De-AC05-00OR22725.

Research sponsored by the Laboratory Directed Research and Development Program of Oak Ridge National Laboratory (ORNL), managed by UT-Battelle, LLC for the U. S. Department of Energy under Contract No. De-AC05-00OR22725.

\section{References}

[1] Cory Ahrens and Gregory Beylkin. Rotationally invariant quadratures for the sphere. Proceedings of the Royal Society of London A: Mathematical, Physical and Engineering Sciences, 465(2110):3103-3125, 2009. 
[2] G. W. Alldredge, C. D. Hauck, and A. L. Tits. High-order entropy-based closures for linear transport in slab geometry II: A computational study of the optimization problem. SIAM J. Sci. Comput., 34(4):B361-B391, 2012.

[3] Graham W. Alldredge, Cory D. Hauck, Dianne P. O'Leary, and André L. Tits. Adaptive change of basis in entropy-based moment closures for linear kinetic equations. Journal of Computational Physics, 258(0):489 - 508, 2014.

[4] Kendall Atkinson. Numerical integration on the sphere. J. Austral. Math. Soc. Ser. B, 23:332-347, 1982.

[5] Kendall Atkinson and Weimin Han. Spherical Harmonics and Approximations on the Unit Sphere: An Introduction. Springer-Verlag, Berlin Heidelberg, 2012.

[6] C. Berthon, M. Frank, C. Sarazin, and R. Turpault. Numerical methods for balance laws with space dependent flux: Application to radiotherapy dose calculation. Communications in Computational Physics, 10(5):1184, 2011.

[7] Thomas A. Brunner. Forms of approximate radiation transport. Technical Report SAND2002-1778, Sandia National Laboratories, 2002.

[8] Thomas A. Brunner and James Paul Holloway. One-dimensional Riemann solvers and the maximum entropy closure. J. Quant Spect. and Radiative Trans, 69(5):543 - 566, 2001.

[9] Jean-François Coulombel, François Golse, and Thierry Goudon. Diffusion approximation and entropy-based moment closure for kinetic equations. Asymptotic Analysis, 45(1):1-39, 2005.

[10] Raúl E. Curto and Lawrence A. Fialkow. Recursiveness, positivity and truncated moment problems. Houston Journal of Mathematics, 4:603-635, 1991.

[11] B. Davison. Neutron Transport Theory. Clarendon Press, Oxford, England, 1957.

[12] S. M. Deshpande. Kinetic theory based new upwind methods for inviscid compressible flows. In American Institute of Aeronautics and Astronautics, New York, 1986. Paper 86-0275.

[13] B. Dubroca and J.-L. Feugeas. Étude théorique et numérique d'une hiérarchie de modèles aux moments pour le transfert radiatif. C.R. Acad. Sci. Paris, I. 329:915-920, 1999.

[14] B Dubroca, J-L Feugeas, and M Frank. Angular moment model for the fokker-planck equation. The European Physical Journal D, 60(2):301-307, 2010.

[15] R. Duclous, B. Dubroca, and M. Frank. A deterministic partial differential equation model for dose calculation in electron radiotherapy. Physics in medicine and biology, 55(13):3843, 2010.

[16] C. Kristopher Garrett and Cory D. Hauck. A comparison of moment closures for linear kinetic transport equations: The line source benchmark. Transport Theory and Statistical Physics, 42(6-7):203-235, 2013.

[17] Thierry Goudon and Chunjin Lin. Analysis of the M1 model: Well-posedness and diffusion asymptotics. Journal of Mathematical Analysis and Applications, 402(2):579-593, 2013.

[18] Clinton Groth and James McDonald. Towards physically realizable and hyperbolic moment closures for kinetic theory. Continuum Mechanics and Thermodynamics, 21(6):467-493, 2009.

[19] A. Harten, P. D. Lax, and Van Leer. On upstream differencing and Godunov-type schemes for hyperbolic conservation laws. SIAM Rev., 25:35-61, 1983.

[20] C. D. Hauck. High-order entropy-based closures for linear transport in slab geometry. Comm. Math. Sci., 9:187-205, 2011.

[21] Cory D. Hauck, C. David Levermore, and André L. Tits. Convex duality and entropy-based moment closures: Characterizing degenerate densities. SIAM J. Control Optim., 47(4):1977-2015, 2008.

[22] Herbert H.H. Homeier and E. Otto Steinborn. Some properties of the coupling coefficients of real spherical harmonics and their relation to Gaunt coefficients. Journal of Molecular Structure: THEOCHEM, 368(0):31 37, 1996. Proceedings of the Second Electronic Computational Chemistry Conference.

[23] M. Junk. Maximum entropy for reduced moment problems. Math. Models Meth. Appl. Sci., 10(7):1001-1025, 2000.

[24] Samuel Karlin and Lloyd S Shapley. Geometry and Moment Spaces. RAND Corporation, 1952.

[25] Jean B Lasserre. Semidefinite programming for gradient and hessian computation in maximum entropy estimation. In Decision and Control, 2007 46th IEEE Conference on, pages 3060-3064. IEEE, 2007.

[26] V.I. Lebedev. Quadratures on a sphere. USSR Computational Mathematics and Mathematical Physics, 16(2):10 $-24,1976$. 
[27] VI Lebedev. Spherical quadrature formulas exact to orders 25-29. Siberian Mathematical Journal, 18(1):99-107, 1977.

[28] V.I. Lebedev. A quadrature formula for the sphere of 59th algebraic order of accuracy. Russian Acad. Sci. Dokl. Math., 50:283-286, 1995.

[29] VI Lebedev and DN Laikov. A quadrature formula for the sphere of the 131st algebraic order of accuracy. In Doklady. Mathematics, volume 59, pages 477-481. MAIK Nauka/Interperiodica, 1999.

[30] V.I. Lebedev and A.L. Skorokhodov. Quadrature formulas of orders 41, 47, and 53 for the sphere. Russian Acad. Sci. Dokl. Math., 45:587-592, 1992.

[31] C. D. Levermore. Moment closure hierarchies for kinetic theory. J. Stat. Phys., 83:1021-1065, 1996.

[32] E. E. Lewis and W. F. Miller, Jr. Computational Methods of Neutron Transport. John Wiley and Sons, New York, 1984.

[33] M. González, E. Audit, and P. Huynh. Heracles: a three-dimensional radiation hydrodynamics code. Astronomy E Astrophysics, 464(2):429-435, 2007.

[34] James McDonald and Manuel Torrilhon. Affordable robust moment closures for CFD based on the maximumentropy hierarchy. Journal of Computational Physics, 251(0):500 - 523, 2013.

[35] Philipp Monreal and Martin Frank. Higher order minimum entropy approximations in radiative transfer. arXiv preprint arXiv:0812.3063, 2008.

[36] Ph. Nicolaï, J.-L. Feugeas, C. Regan, M. Olazabal-Loumé, J. Breil, B. Dubroca, J.-P. Morreeuw, and V. Tikhonchuk. Effect of the plasma-generated magnetic field on relativistic electron transport. Phys. Rev. E, 84:016402, Jul 2011.

[37] Department of Energy Scientific Grand Challenges Workshop Series. Architectures and technology for extreme scale computing, 2009.

[38] B. Perthame. Boltzmann type schemes for gas dynamics and the entropy property. SIAM J. on Numer. Anal., 27(6):1405-1421, 1990.

[39] B. Perthame. Second-order Boltzmann schemes for compressible Euler equations in one and two space dimensions. SIAM J. Numer. Anal., 29(1):1-19, 1992.

[40] G. C. Pomraning. The Equations of Radiation Hydrodynamics. Pergamon Press, New York, 1973.

[41] J. Rasch and A. Yu. Efficient storage scheme for precalculated Wigner 3j, 6j and Gaunt coefficients. SIAM Journal on Scientific Computing, 25(4):1416-1428, 2004.

[42] Yousef Saad. Iterative Methods for Sparse Linear Systems. Society for Industrial and Applied Mathematics, Philadelphia, PA, second edition, 2003.

[43] J. Schneider. Entropic approximation in kinetic theory. Math. Model. Numer. Anal., 38:541-561, 2004.

[44] James Alexander Shohat and Jacob David Tamarkin. The Problem of Moments. American Mathematical Society, New York, 1943.

[45] R. Turpault. A multigroup M1 model for radiation hydrodynamics and applications. In A.D. Ketsdever and E.P. Muntz, editors, 23rd internationnal symposium on rarefied gas dynamics. American Institute of Physics, 2004.

[46] W. Walters. Use of the Chebyshev-Legendre quadrature set in discrete-ordinate codes. Technical Report LAUR-87-3621, Los Alamos National Laboratory, 1987.

[47] D. Wright, M. Frank, and A. Klar. The minimum entropy approximation to the radiative transfer equation. Proc. Symp. Appl. Math., 67:987-996, 2009. 